\title{
HETEROGENEOUS MONOID AUTOMATA AND ADMISSIBLE BISYSTEMS
}

\author{
H.-J. H O E H N K E
}

Institute of Mathematics, Academy of Sciences of the German Democratic Republic, Berlin, G.D.R.

\section{Introduction}

Bainbridge [1] introduced the characteristic bisystem char $(S)$ of a sequential automaton $(S)$ with say $n$ input and $k$ output alphabets in such a manner that the space $C$ of configurations of $(S)$ is formed and then is established a certain congruence in $C$ taking into account the structure of $(S)$.

In the present paper we directly (without forming a congruence) construct char $(S)$ by means of a change to a corresponding monoid automaton $\boldsymbol{F}(\boldsymbol{S})$ and then forming the bisystem of the compositum $\varepsilon(\boldsymbol{X})$ $F(S) \mu(\boldsymbol{Y})$ (cf. (2.5)). Since it is hard to get an insight into the preservation properties of char $(S)$ we introduce the new concepts of a heterogeneous monoid automaton and a heterogeneous (admissible) bisystem using general coding maps $\varepsilon, \mu, \nu$, which may be of an independent interest. Nevertheless even then preservation properties are difficult to establish (cf. Theorem 4.2).

Our study is a contribution to application of algebraic methods in the study of the behaviour of sequential automata with several inputs and several outputs. The study of loop operation (feedback) is postponed to another occasion.

\section{Sequential automata}

A sequential automaton in the category set of all sets and all mappings between sets is a quintuple

$$
\langle A, B, S, l: S \times A \rightarrow B, m: S \times A \rightarrow S\rangle
$$


written also as $S \stackrel{l}{\leftarrow} S \times A \stackrel{m}{\rightarrow} B$ or $A \stackrel{S}{\rightarrow, m} B$ or for short $S: A \rightarrow B$, where $A, B, S$ are the input set, the output set, and the set of states respectively; $l$ is the input-output map and $m$ is the next-state or transition map.

Serial connection (.) of automata $S: A \rightarrow B, S^{\prime}: B \rightarrow C$ is defined according to

$$
(A \underset{l, m}{\stackrel{S}{\longrightarrow}} B)\left(B \underset{l^{\prime}, m^{\prime}}{\stackrel{S^{\prime}}{\longrightarrow}} C\right)=A \underset{l^{\prime \prime}, m^{\prime \prime}}{S^{\prime} \times S} C
$$

where $l^{\prime \prime}$ and $m^{\prime \prime}$ are given by the following defining commutative diagrams (with $d_{R}$ for any set $R$ denoting the diagonal map

$$
\left.d_{R}: R \rightarrow R \times R: r \mapsto(r, r)\right):
$$

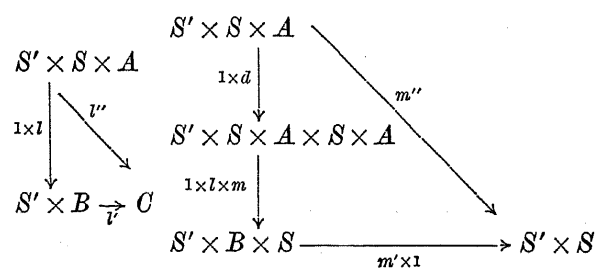

(Here for short we write $1 \times d$ instead, of $1_{S^{\prime}} \times d_{S \times A}$, where $1_{S^{\prime}}$ denotes the identity map $S^{\prime} \rightarrow S^{\prime}$, and so on.)

With respect to serial connection as the composition the sequential automata in Set form a category Set-Aut $\rightarrow$ under the assumption that the Cartesian product $(x)$ between sets approximately is considered as strictly associative.

Parallel connection of automata $S: A \rightarrow B, S^{\prime}: A^{\prime} \rightarrow B^{\prime}$ (also denoted by $\times$ ) is defined according to

$$
(A \underset{l, m}{\stackrel{S}{\longrightarrow}} B) \times\left(A^{\prime} \underset{l, m}{\stackrel{S}{\longrightarrow}} B^{\prime}\right)=A \times A^{\prime} \underset{l^{\prime \prime}, m^{\prime \prime}}{\stackrel{S \times S^{\prime \prime}}{\longrightarrow}} B \times B^{\prime},
$$

where $l^{\prime \prime}$ and $m^{\prime \prime}$ are given by the following defining commutative diagram (with $c_{S^{\prime} A}$ standing for the symmetry map

$$
\begin{aligned}
& \left.c_{S^{\prime} \Lambda}: S^{\prime} \times A \rightarrow A \times S^{\prime}:\left(s^{\prime}, a\right) \mapsto\left(a, s^{\prime}\right)\right): \\
& S \times S^{\prime} \stackrel{m^{\prime \prime}}{\longleftarrow} S \times S^{\prime} \times A \times A^{\prime} \stackrel{l^{\prime \prime}}{\longrightarrow} B \times B^{\prime}
\end{aligned}
$$

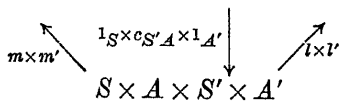

Monoid automata are sequential automata with an additional structure. A monoid automaton in Set in the sense of $R$. C. Davis and the author (cf. Budach-Hoehnke [2]) is a sequential automaton $A \underset{l, m}{\rightarrow} B$, where $\left\langle A,\left({ }^{A}\right), 1^{A}\right\rangle$ and $\left\langle B,\left({ }^{B}\right), 1^{B}\right\rangle$ are monoids, also for short denoted by $A$ and $B$ respectively, and $\langle S, m\rangle$ is a unital $A$-system, i.e., the diagrams

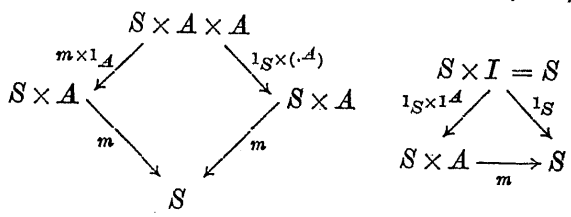

are commutative, where $I$ is the one element set $I=\{\varnothing\}$, and $1^{\mathcal{1}}$ is at the same time considered as the map

$$
1^{A}: I \rightarrow A: \emptyset \mapsto 1^{A} .
$$

Moreover, the commutativity of the following diagrams is required (with $t_{S}$ as the terminal map

$$
\left.t_{S}: S \rightarrow I: s \mapsto \varnothing\right):
$$

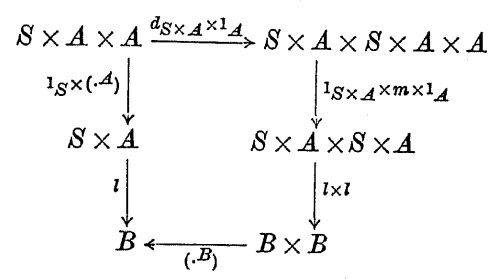

$$
S \times I=S \stackrel{1^{I_{S} \times 1^{A}}}{\longrightarrow} S \times
$$

For short we write $l(s, a)=s \circ a, m(s, a)=s . a$.

Serial and parallel connections of two monoid automata are defined as in the case of ordinary sequential automata. As proved in BudachHoehnke ([2], p. 258), they again are monoid automata. The category of all monoid automata in Set with serial connection as composition is denoted by Set-Maut .

Let $X$ be a set and let $X^{*}$ be the free monoid freely generated by $X$. To any sequential automaton $X \underset{l, m}{\rightarrow} Y$ we assign a new sequential automaton $X^{*} \underset{O,}{\rightarrow} Y^{*}$ with the inductively defined maps:

(*): $S \times X^{*} \rightarrow X^{*}$,

$$
\begin{gathered}
s \cdot\left(x_{1} x_{2} \ldots x_{n}\right)=m\left(s \cdot\left(x_{1} x_{2} \ldots x_{n-1}\right), x_{n}\right), \\
s \cdot 1^{X^{*}}=s,
\end{gathered}
$$


(o): $S \times X^{*} \rightarrow Z^{*}$

$$
\begin{aligned}
s \circ\left(x_{1} x_{2} \ldots x_{n}\right)= & \left(s \circ\left(x_{1} x_{2} \ldots x_{n-1}\right)\right) l\left(s \cdot\left(x_{1} x_{2} \ldots x_{n-1}\right), x_{n}\right), \\
& s \circ 1^{X^{*}}=1^{T^{*}} .
\end{aligned}
$$

In Budach-Hoehnke ([2], pp. 257, 265) it was proved:

LEMMa 1.1. $X^{*} \underset{0, S^{\circ}}{\longrightarrow} Y^{*}$ is a monoid automaton and the assignment

$$
X \underset{l, m}{\stackrel{s}{\longrightarrow}} Y \mapsto X^{*} \underset{\mathrm{O}^{\circ}}{\rightarrow} Y^{*}
$$

defines a functor

$$
F: \text { Set-Aut } \rightarrow \text { Set-Maut } \rightarrow
$$

We consider $\left(X_{1} \times \ldots \times X_{n}\right)^{*}$ (where $X_{1}, \ldots, X_{n}$ are sets) as a submonoid of $X_{1}^{*} \times \ldots \times X_{n}^{*}$ according to the embedding

$$
\left(X_{1} \times \ldots \times X_{n}\right)^{*} \stackrel{\varepsilon}{\longrightarrow} X_{1}^{*} \times \ldots \times X_{n}^{*}
$$

received by the equality $v=v^{1} \ldots v^{k}=\left\langle v_{1}, \ldots, v_{k}\right\rangle$, where

and

$$
v^{1}=\left\langle x_{1}^{1}, \ldots, x_{n}^{1}\right\rangle, \ldots, v^{k}=\left\langle x_{1}^{k}, \ldots, x_{n}^{k}\right\rangle \in X_{1} \times \ldots \times X_{n}
$$

$$
v_{1}=x_{1}^{1} \ldots x_{1}^{k} \in X_{1}^{*}, \quad \ldots, \quad v_{n}=x_{n}^{1} \ldots x_{n}^{k} \in X_{n}^{*} .
$$

For short we introduce the following notation. If $\boldsymbol{X}=\left\langle X_{1}, \ldots, X_{n}\right\rangle$ is an $n$-tuple of sets $X_{i}$, then $\times \boldsymbol{X}=X_{1} \times \ldots \times X_{n}$. So, if $\boldsymbol{X}^{*}=\left\langle X_{1}^{*}, \ldots\right.$ $\left.\ldots, X_{n}^{*}\right\rangle$, then $\times X^{*}=X_{1}^{*} \times \ldots \times X_{n}^{*}$. The inclusion map $(\subseteq):(\times X)^{*}$ $\rightarrow \times \boldsymbol{X}^{*}$ defined above gives rise to a monoid automaton

$$
\mu(\boldsymbol{X})=(\times \boldsymbol{X})^{*} \underset{(\mathcal{S}), t(\times \boldsymbol{X})^{*}}{\longrightarrow} \times \boldsymbol{X}^{*} .
$$
holds

LEMArA 1.2. Let $X \underset{l, m}{\stackrel{s}{\longrightarrow}} Y, X^{\prime} \underset{l^{\prime}, m^{\prime}}{\stackrel{s^{\prime}}{\longrightarrow}} Y^{\prime}$ be sequential automata. Then it $F\left((X \underset{l, m}{\stackrel{s}{\longrightarrow}} Y) \times\left(X^{\prime} \frac{s^{\prime}}{l^{\prime}, m^{\prime}} Y^{\prime}\right)\right) \mu\left(Y, Y^{\prime}\right)$

Proof. Let be

$$
=\mu\left(X, X^{\prime}\right)\left(F\left(X \underset{l, m^{\prime}}{\stackrel{s}{\longrightarrow}} Y\right) \times F^{\prime}\left(X^{\prime} \underset{l^{\prime}, m^{\prime}}{\stackrel{s^{\prime}}{\longrightarrow}} X^{\prime}\right)\right) .
$$

and

$$
(X \underset{l, m}{\stackrel{s}{\longrightarrow}} Y) \times\left(X^{\prime} \underset{l^{\prime}, m^{\prime}}{\stackrel{s^{\prime}}{\longrightarrow}} Y^{\prime}\right)=X \times X^{\prime} \underset{l^{\prime \prime}, m^{\prime \prime}}{\stackrel{S \times S^{\prime}}{\longrightarrow}} Y \times Y^{\prime}
$$

$$
\begin{aligned}
& F(X \underset{l, m}{\stackrel{s}{\longrightarrow}} Y)=X^{*} \underset{0, .}{\stackrel{s}{\longrightarrow}} Y^{*}, \quad F\left(X^{\prime} \underset{l^{\prime}, m^{\prime}}{\stackrel{S^{\prime}}{\rightarrow}} Y^{\prime}\right)=X^{\prime *} \underset{0^{\prime},,^{\prime \prime}}{\stackrel{S^{\prime}}{\rightarrow}} Y^{\prime *}, \\
& F\left(X \times X^{\prime} \frac{S \times S^{\prime}}{l^{\prime \prime, m^{\prime \prime}}} Y \times Y^{\prime}\right)=\left(X \times X^{\prime}\right)^{*} \frac{S \times S^{\prime}}{0^{*}, * *}\left(Y \times Y^{\prime}\right)^{*} .
\end{aligned}
$$

Then by induction

$$
\begin{aligned}
\left(s, s^{\prime}\right) \circ^{*}\left(\left(x_{1}, x_{1}^{\prime}\right) \ldots\left(x_{n}, x_{n}^{\prime}\right)\right) & =\left(s \circ\left(x_{1} \ldots x_{n}\right), s^{\prime} \circ^{\prime}\left(x_{1}^{\prime} \ldots x_{n}^{\prime}\right)\right), \\
\left(s, s^{\prime}\right) \bullet^{*}\left(\left(x_{1}, x_{1}^{\prime}\right) \ldots\left(x_{n}, x_{n}^{\prime}\right)\right) & =\left(s \cdot\left(x_{1} \ldots x_{n}\right), s^{\prime} \cdot{ }^{\prime}\left(x_{1}^{\prime} \ldots x_{n}^{\prime}\right)\right) .
\end{aligned}
$$

We consider the sequential automaton depending upon $\boldsymbol{X}=\left\langle X_{1} \ldots, X_{n}\right\rangle$

$$
\varepsilon(\boldsymbol{X})=\times \boldsymbol{X}^{*} \underset{i_{n=1}, m_{n}}{\stackrel{n}{u} \tilde{x}_{i}} \longrightarrow(X \boldsymbol{X})^{*},
$$

where $\bigcup_{i=1}^{n} \dot{X}_{i}$ is the union of the monoids

$$
\tilde{X}_{i}=X_{1}^{*} \times \ldots \times X_{i-1}^{*} \times\left\{1^{X_{i}^{*}}\right\} \times X_{i+1}^{*} \times \ldots \times X_{n}^{*},
$$

with the intersections

$$
\begin{aligned}
\tilde{X}_{i} \cap \tilde{X}_{j}=X_{1}^{*} \times \ldots \times X_{i-1}^{*} \times\left\{1^{x_{i}^{*}}\right\} \times X_{i+1}^{*} \times \ldots \times X_{j-1}^{*} \times\left\{1^{x_{j}^{*}}\right\} \times \\
\times X_{j+1}^{*} \times \ldots \times X_{n}^{*} \quad \text { for } i<j,
\end{aligned}
$$

and where the definition of $l_{n}$ and $m_{n}$ is derived by means of the unique decomposition of the product $u w$ of word sequences

$$
u=\left\langle u_{1}, \ldots, u_{i-1}, 1, u_{i+1}, \ldots, u_{n}\right\rangle \in \tilde{X}_{i}, \quad w=\left\langle w_{1}, \ldots, w_{n}\right\rangle \in \times \boldsymbol{X}^{*}
$$

into the product $v u^{\prime}$ of elements

$$
v=v^{1} \ldots v^{k} \in(\times \boldsymbol{X})^{*}, \quad u^{\prime}=\left\langle u_{1}^{\prime}, \ldots, u_{n}^{\prime}\right\rangle \in \bigcup_{i=1}^{n} \tilde{X}_{i}
$$

(hence it exists at least one index $j$ for which $u_{j}^{\prime}=1 \in X_{j}^{*}$ ) such that

and therefore

$$
l_{n}(u, w)=v, \quad m_{n}(u, w)=u^{\prime}
$$

The importance of $\boldsymbol{\varepsilon}(\boldsymbol{X})$ for our further considerations mainly rests upon:

LEMMA 1.3. $\varepsilon(\boldsymbol{X})$ is a monoid automaton in Set. we get

Proof. Let be $w, w^{\prime} \in \boldsymbol{X}$. Taking into consideration (1.1) similarly

$$
\begin{aligned}
l_{n}\left(u, w w^{\prime}\right) m_{n}\left(u, w w^{\prime}\right) & =u\left(w w^{\prime}\right)=(u w) w^{\prime}=l_{n}(u, w) m_{n}(u, w) w^{\prime} \\
& =l_{n}(u, w) l_{n}\left(m_{n}(u, w), w^{\prime}\right) m_{n}\left(m_{n}(u, w), w^{\prime}\right),
\end{aligned}
$$

hence because of the uniqueness of the decomposition,

$l_{n}\left(u, w w^{\prime}\right)=l_{n}(u, w) l_{n}\left(m_{n}(u, w), w^{\prime}\right), \quad m_{n}\left(u, w w^{\prime}\right)=m_{n}\left(m_{n}(u, w), w^{\prime}\right)$. 
Since $u=u 1=l_{n}(u, 1) m_{n}(u, 1)$ we get

$$
l_{n}(u, 1)=1, \quad m_{n}(u, 1)=u .
$$

If $\underline{\boldsymbol{X}}=\left\langle\boldsymbol{X}^{\mathrm{I}}, \ldots, \boldsymbol{X}^{n}\right\rangle$ is a sequence of length $n$ of sequences

$$
\boldsymbol{X}^{1}=\left\langle X_{1}^{1}, \ldots, X_{n_{1}}^{1}\right\rangle, \quad \ldots, \quad \boldsymbol{X}^{n}=\left\langle X_{1}^{n}, \ldots, X_{n_{n}}^{n}\right\rangle
$$

of sets $X_{j}^{i}$ we use the notation

$$
[\underline{\boldsymbol{X}}]=\left[\boldsymbol{X}^{1}, \ldots, \boldsymbol{X}^{n}\right]=\left\langle X_{1}^{1}, \ldots, X_{n_{1}}^{1}, \ldots, X_{1}^{n}, \ldots, X_{n_{n}}^{n}\right\rangle
$$

forgetting the subdivision of this sequence of length $n_{1}+\ldots+n_{n}$. This notation is ambiguous in the case $n=1$ and then only applied in a suitable context.

LEMTMA 1.4. If $X_{1}, \ldots, X_{n}, X_{1}^{\prime}, \ldots, X_{n^{\prime}}^{\prime}$, are sets and $\boldsymbol{X}=\left\langle X_{1}, \ldots\right.$ $\left.\ldots, X_{n}\right\rangle, \boldsymbol{X}^{\prime}=\left\langle X_{1}^{\prime}, \ldots, X_{n^{\prime}}^{\prime}\right\rangle$, then $\varepsilon\left(\left[\boldsymbol{X}, \boldsymbol{X}^{\prime}\right]\right)$ decomposes up to isomorphy according to

$$
\left(\varepsilon(\boldsymbol{X}) \times \varepsilon\left(\boldsymbol{X}^{\prime}\right)\right) \varepsilon\left(\times \boldsymbol{X}, \times \boldsymbol{X}^{\prime}\right) \stackrel{\cong}{\longrightarrow} \varepsilon\left(\left[\boldsymbol{X}, \boldsymbol{X}^{\prime}\right]\right) .
$$

Proof. We form

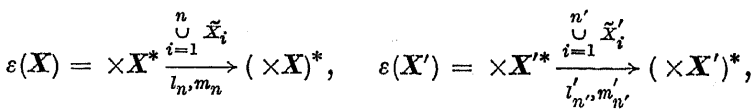

$$
\begin{aligned}
& \varepsilon\left(\left[\boldsymbol{X}, \boldsymbol{X}^{\prime}\right]\right)=\times\left[\boldsymbol{X}, \boldsymbol{X}^{\prime}\right]^{*} \underset{l^{\prime \prime}, m^{\prime \prime}}{\stackrel{n+n^{\prime}}{\underbrace{\prime \prime}_{i=1}} \tilde{x}_{i}^{\prime \prime}}\left(\times\left[\boldsymbol{X}, \boldsymbol{X}^{\prime}\right]\right)^{*},
\end{aligned}
$$

where

$$
\begin{aligned}
\bigcup_{i=1}^{n+n^{\prime}} \tilde{X}_{i}^{\prime \prime} & =\bigcup_{i=1}^{n} \tilde{X}_{i} \times\left(\times \boldsymbol{X}^{\prime *}\right) \cup\left(\times \boldsymbol{X}^{*}\right) \times \bigcup_{i=1}^{n^{\prime}} \tilde{X}_{i}^{\prime} \\
& =\left(\bigcup_{i=1}^{n} \tilde{X}_{i} \times\left(\left(\times \boldsymbol{X}^{\prime}\right)^{*} \cdot \bigcup_{i=1}^{n^{\prime}} \tilde{X}_{i}^{\prime}\right)\right) \cup\left(\left((\times \boldsymbol{X})^{*} \cdot \bigcup_{i=1}^{n} \tilde{X}_{i}\right) \times \bigcup_{i=1}^{n^{\prime}} \tilde{X}_{i}^{\prime}\right) \\
& =\left((\times \boldsymbol{X})^{*} \times\left\{1^{\left(\times \boldsymbol{X}^{\prime}\right)^{*}}\right\} \cup\left\{1^{\left(\times X^{*}\right.}\right\} \times\left(\times \boldsymbol{X}^{\prime}\right)^{*}\right) \cdot\left(\left(\bigcup_{i=1}^{n} \tilde{X}_{i}\right) \times\left(\bigcup_{i=1}^{n^{\prime}} \tilde{X}_{i}^{\prime}\right)\right) .
\end{aligned}
$$

With respect to this decomposition we define a map

for

$$
e: S \rightarrow \bigcup_{i=1}^{n+n^{\prime}} \tilde{X}_{i}^{\prime \prime}
$$

$$
S=\left((\times \boldsymbol{X})^{*} \times\left\{1^{\left(\times X^{\prime}\right)^{*}}\right\} \cup\left\{1^{(\times X) *}\right\} \times\left(\times \boldsymbol{X}^{\prime}\right)^{*}\right) \times\left(\bigcup_{i=1}^{n} \tilde{X}_{i}\right) \times\left(\bigcup_{i=1}^{n^{\prime}} \tilde{X}_{i}^{\prime}\right)
$$

by

$$
\begin{aligned}
& \left\langle v, u, u^{\prime}\right\rangle \mapsto\left\langle v u, u^{\prime}\right\rangle \quad \text { if } \quad v \in(\times \boldsymbol{X})^{*}, \\
& \left\langle v^{\prime}, u, u^{\prime}\right\rangle \mapsto\left\langle u, v^{\prime} u^{\prime}\right\rangle \quad \text { if } \quad v^{\prime} \in\left(\times \boldsymbol{X}^{\prime}\right)^{*} .
\end{aligned}
$$

It is clear that $e$ is one-to-one and onto.

Using the symmetry

$$
e=c_{U V} \quad \text { for } \quad U=\bigcup_{i=1}^{n^{\prime}} \tilde{X}_{i}^{\prime}, \quad \nabla=\times X^{*}
$$

and the diagonal

$$
d=d_{W} \quad \text { for } \quad W=\left(\left(\bigcup_{i=1}^{n} \tilde{X}_{i}\right) \times\left(\bigcup_{i=1}^{n^{\prime}} \tilde{X}_{i}^{\prime}\right)\right) \times\left(\left(\times \boldsymbol{X}^{*}\right) \times\left(\times \boldsymbol{X}^{\prime *}\right)\right)
$$

we have

$$
\begin{aligned}
& \left(\varepsilon(\boldsymbol{X}) \times \varepsilon\left(\boldsymbol{X}^{\prime}\right)\right) \varepsilon\left(\times \boldsymbol{X}, \times \boldsymbol{X}^{\prime}\right)
\end{aligned}
$$

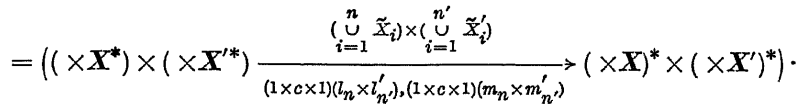

$$
\begin{aligned}
& \cdot\left((\times \boldsymbol{X})^{*} \times\left(\times \boldsymbol{X}^{\prime}\right)^{*} \underset{(\times \boldsymbol{X})^{*} \cup(\times \boldsymbol{X})^{*}}{l_{2}^{\times}, m_{2}^{\times}} \rightarrow\left(\times\left[\boldsymbol{X}, \boldsymbol{X}^{\prime}\right]\right)^{*}\right) \\
& =\left(\times \boldsymbol{X}^{*}\right) \times\left(\times \boldsymbol{X}^{\prime *}\right) \underset{l, m}{\stackrel{S}{\longrightarrow}}\left(\times\left[\boldsymbol{X}, \boldsymbol{X}^{\prime}\right]\right)^{*}
\end{aligned}
$$

and for $u \in(\times \boldsymbol{X})^{*} \times\left\{1^{\left(\times X^{\prime}\right) *}\right\} \cup\left\{1^{(\times X)^{*}}\right\} \times\left(\times \boldsymbol{X}^{\prime}\right)^{*}$ (for short to be identified with $\left.(X \boldsymbol{X})^{*} \cup\left(X \boldsymbol{X}^{\prime}\right)^{*}\right)$

$$
u\left\langle w, w^{\prime}\right\rangle=l_{2}^{\times}\left(u,\left\langle w, w^{\prime}\right\rangle\right) m_{2}^{\times}\left(u,\left\langle w, w^{\prime}\right\rangle\right)
$$

and $\left(\right.$ with $d^{\prime}=d_{W^{\prime}}$ for $\left.W^{\prime}=\left(\bigcup_{i=1}^{n} \tilde{X}_{i}\right) \times\left(\times \boldsymbol{X}^{*}\right) \times\left(\bigcup_{i=1}^{n^{\prime}} \tilde{X}_{i}^{\prime}\right) \times\left(\times \boldsymbol{X}^{\prime *}\right)\right)$

$$
l=\left(1 \times(1 \times c \times 1)\left(l_{n} \times l_{n^{\prime}}^{\prime}\right)\right) l_{2}^{\times},
$$

$$
\begin{aligned}
m & =(1 \times d)\left(1 \times((1 \times c \times 1) \times(1 \times c \times 1))\left(l_{n} \times l_{n^{\prime}}^{\prime} \times m_{n} \times m_{n^{\prime}}^{\prime}\right)\right)\left(m_{2}^{\times} \times 1\right) \\
& =\left(1 \times(1 \times c \times 1) d^{\prime}\left(l_{n} \times l_{n^{\prime}}^{\prime} \times m_{n} \times m_{n^{\prime}}^{\prime}\right)\right)\left(m_{2}^{\times} \times 1\right) .
\end{aligned}
$$

Using the equality

(1.3) $\quad u^{\prime \prime} w^{\prime \prime}=l^{\prime \prime}\left(u^{\prime \prime}, w^{\prime \prime}\right) m^{\prime \prime}\left(u^{\prime \prime}, w^{\prime \prime}\right)$ for $u^{\prime \prime} \in \bigcup_{i=1}^{n+n^{\prime}} \tilde{X}_{i}, w^{\prime \prime} \in \times\left[\boldsymbol{X}, \boldsymbol{X}^{\prime}\right]^{*}$

23 - Banach Center Publ. t. 9 
we are able to verify that $\left\langle 1_{\times\left[\boldsymbol{x}, \boldsymbol{x}^{*}\right]^{*}}, 1_{\left(\mathbf{x}\left[\boldsymbol{x}, \boldsymbol{x}^{\prime}\right)^{*}\right.}, e\right\rangle$ is an isomorphism of monoid automata in Set in the sense of the author, cf. Budach-Hoehnke ([2], p. 249), i.e., that the following diagram is commutative.

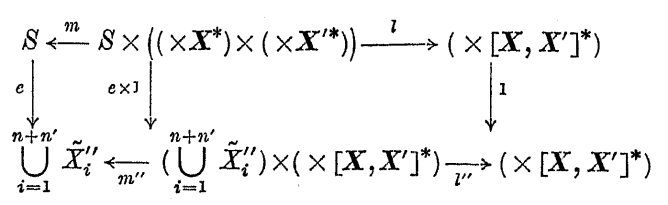

In fact for $v \in(X \boldsymbol{X})^{*}, \cup\left(\times \boldsymbol{X}^{\prime}\right)^{*}$ we get

$$
\begin{aligned}
& \left\langle v,\left\langle u, u^{\prime}\right\rangle,\left\langle w, w^{\prime}\right\rangle\right\rangle \stackrel{e \times 1}{\longrightarrow}\left\langle v\left\langle u, u^{\prime}\right\rangle,\left\langle w, w^{\prime}\right\rangle\right\rangle \mid \stackrel{l^{\prime \prime}}{\longrightarrow} l^{\prime \prime}\left(v\left\langle u, u^{\prime}\right\rangle,\left\langle w, w^{\prime}\right\rangle\right), \\
& \left\langle v,\left\langle u, u^{\prime}\right\rangle,\left\langle w, w^{\prime}\right\rangle\right\rangle\left|\stackrel{e \times 1}{\longrightarrow}\left\langle v\left\langle u, u^{\prime}\right\rangle,\left\langle w, w^{\prime}\right\rangle\right\rangle\right| \stackrel{m^{\prime \prime}}{\longrightarrow} m^{\prime \prime}\left(v\left\langle u, u^{\prime}\right\rangle,\left\langle w, w^{\prime}\right\rangle\right) \text {, } \\
& v\left\langle u, u^{\prime}\right\rangle\left\langle w, w^{\prime}\right\rangle=v\left\langle u w, u^{\prime} w^{\prime}\right\rangle \\
& =l^{\prime \prime}\left(v\left\langle u, u^{\prime}\right\rangle,\left\langle w, w^{\prime}\right\rangle\right) m^{\prime \prime}\left(v\left\langle u, u^{\prime}\right\rangle,\left\langle w, w^{\prime}\right\rangle\right), \\
& \left\langle v,\left\langle u, u^{\prime}\right\rangle,\left\langle w, w^{\prime}\right\rangle\right\rangle \stackrel{1 \times(1 \times c \times 1)}{\longrightarrow}\left\langle v, u, w, u^{\prime}, w^{\prime}\right\rangle \\
& \stackrel{1 \times\left(l_{n} \times l_{n}^{\prime}\right)}{\longrightarrow}\left\langle v, l_{n}(u, w), l_{n^{\prime}}^{\prime}\left(u^{\prime}, w^{\prime}\right)\right\rangle \stackrel{l_{2}^{\times}}{\longrightarrow} l_{2}^{\times}\left(v,\left\langle l_{n}(u, w), l_{n^{\prime}}^{\prime}\left(u^{\prime}, w^{\prime}\right)\right\rangle\right), \\
& \left\langle v,\left\langle u, u^{\prime}\right\rangle,\left\langle w, w^{\prime}\right\rangle\right\rangle \mid \stackrel{1 \times(1 \times c \times 1)}{\longrightarrow}\left\langle v, u, w, u^{\prime}, w^{\prime}\right\rangle \\
& \stackrel{1 \times a}{\longrightarrow}\left\langle v, u, w, u^{\prime}, w^{\prime}, u, w, u^{\prime}, w^{\prime}\right\rangle \\
& \stackrel{1 \times\left(l_{n} \times l_{n^{\prime}}^{\prime} \times m_{n} \times m_{n^{\prime}}^{\prime}\right)}{\longrightarrow}\left\langle v, l_{n}(u, w), l_{n^{\prime}}^{\prime}\left(u^{\prime}, w^{\prime}\right), m_{n}(u, w), m_{n^{\prime}}^{\prime}\left(u^{\prime}, w^{\prime}\right)\right\rangle \\
& \stackrel{m_{2}^{\times} \times 1}{\longrightarrow}\left\langle m_{2}^{\times}\left(v, l_{n}(u, w), l_{n^{\prime}}^{\prime}\left(u^{\prime}, w^{\prime}\right)\right), m_{n}(u, w), m_{n^{\prime}}^{\prime}\left(u^{\prime}, w^{\prime}\right)\right\rangle \\
& \stackrel{e}{\longrightarrow} m_{2}^{\times}\left(v, l_{n}(u, w), l_{n^{\prime}}^{\prime}\left(u^{\prime}, w^{\prime}\right) \mid\left\langle m_{n}(u, w), m_{n^{\prime}}^{\prime}\left(u^{\prime}, w^{\prime}\right)\right\rangle,\right. \\
& v\left\langle l_{n}(u, w), l_{n^{\prime}}^{\prime}\left(u^{\prime}, w^{\prime}\right)\right\rangle \\
& =l_{2}^{\times}\left(v,\left\langle l_{n}(u, w), l_{n^{\prime}}^{\prime}\left(u^{\prime}, w^{\prime}\right)\right\rangle\right) m_{2}^{\times}\left(v,\left\langle l_{n}(u, w), l_{n^{\prime}}^{\prime}\left(u^{\prime}, w^{\prime}\right)\right\rangle\right), \\
& u w=l_{n}(u, w) m_{n}(u, w), \quad u^{\prime} w^{\prime}=l_{n^{\prime}}^{\prime}\left(u^{\prime}, w^{\prime}\right) m_{n^{\prime}}^{\prime}\left(u^{\prime}, w^{\prime}\right),
\end{aligned}
$$

$$
\cdot\left\langle m_{n}(u, w), m_{n^{\prime}}\left(u^{\prime}, w^{\prime}\right)\right\rangle
$$

therefore

$$
\begin{aligned}
l^{\prime \prime}\left(v\left\langle u, u^{\prime}\right\rangle,\langle w, w\rangle\right)=l_{2}^{\times}\left(v,\left\langle l_{n}(u, w), l_{n^{\prime}}^{\prime}\left(u^{\prime}, w^{\prime}\right)\right\rangle\right), \\
m^{\prime \prime}\left(v\left\langle u, u^{\prime}\right\rangle,\left\langle w, w^{\prime}\right\rangle\right) \\
\quad=m_{2}^{\times}\left(v,\left\langle l_{n}(u, w), l_{n^{\prime}}^{\prime}\left(u^{\prime}, w^{\prime}\right)\right\rangle\right)\left\langle m_{n}(u, w), m_{n^{\prime}}^{\prime}\left(u^{\prime}, w^{\prime}\right)\right\rangle .
\end{aligned}
$$

\section{Bisystems}

Let $S$ and $T$ be monoids. A unital $(S, T)$-bisystem $Q$ is a unary algebra $\langle Q, S, T\rangle$ with carrier set $Q$ and with unary operations corresponding to the elements $s \in S, t \in T$ applied to the elements $q \in Q$ from left and from right respectively according to

$$
(s, q) \mapsto s q \in Q, \quad(q, t) \mapsto q t \in Q
$$

such that the following identities hold

$$
\begin{array}{ll}
1^{S} q=q, & \left(s_{1} s_{2}\right) q=s_{1}\left(s_{2} q\right) \quad(Q \text { is a unital } S \text {-left system), } \\
q 1^{T}=q, & q\left(t_{1} t_{2}\right)=\left(q t_{1}\right) t_{2} \quad(Q \text { is a unital } T \text {-rigth system), } \\
& (s q) t=s(q t) \quad \text { ("associativity" law). }
\end{array}
$$

A unital $(S, T)$-bisystem $Q$ is equivalently described as a functor

$$
\begin{aligned}
& \Phi: S^{\text {OD }} \times T \rightarrow \text { Set, } \\
& \left(1^{S}, 1^{T}\right) \mapsto 1_{Q}: Q \rightarrow Q, \\
& (s, t)=\left(s, 1^{T}\right)\left(\mathbb{1}^{S}, t\right)=\left(\mathbb{1}^{S}, t\right)\left(s, 1^{T}\right) \\
& \mapsto \Phi(s, t)=\Phi\left(s, 1^{T}\right) \Phi\left(1^{S}, t\right)=\Phi\left(1^{S}, t\right) \Phi\left(s, 1^{T}\right)=s q t, \\
& \Phi\left(s, \mathcal{1}^{T}\right)(q)=s q, \quad \Phi\left(1^{S}, t\right)(q)=q t .
\end{aligned}
$$

Here the monoids $S$ and $T$ are considered as categories with only one object corresponding to the identity element $1^{S}$ and $1^{T}$ respectively.

LEMNA 2.1. To every monoid automaton $A \underset{l, m}{\stackrel{S}{\rightarrow}} B$ there corresponds a unital $(B, A)$-bisystem $B \times S$ given by the following right and left multiplications applied to $(b, s) \in B \times S$ :

$$
\begin{gathered}
\langle b, s) a=(b l(s, a), m(s, a)) \quad(a \in A), \\
b^{\prime}(b, s)=\left(b^{\prime} b, s\right) \quad\left(b^{\prime} \in B\right) .
\end{gathered}
$$

We denote this unital $(B, A)$-bisystem corresponding to $A \underset{l, m}{S} B$ by $G(A \underset{l, m}{\stackrel{S}{\longrightarrow}} B)$. 
A unital $(B, A)$-bisystem $R$ is called admissible if it satisfies the following conditions:

(i) $R=B \times S$,

(ii) $S$ is a unital $A$-right system,

(iii) $b^{\prime}(b, s)=\left(b^{\prime} b, s\right)$ for all $b, b^{\prime} \in B, s \in S$,

(iv') it exists a function $l: S \times A \rightarrow B$ such that

$$
(b, s) a=(b l(s, a), s a) .
$$

Since $R$ is a $(B, A)$-bisystem, from $\left(\mathrm{iv}^{\prime}\right)$ it follows

$$
\begin{aligned}
(b, s)\left(a_{1} a_{2}\right) & =\left(b l\left(s, a_{1} a_{2}\right), s\left(a_{1} a_{2}\right)\right) \\
& =\left(b l\left(s, a_{1}\right), s a_{1}\right) a_{2} \\
& =\left(b l\left(s, a_{1}\right) l\left(s a_{1}, a_{2}\right),\left(s a_{1}\right) a_{2}\right) \\
& =\left(b l\left(s, a_{1}\right) l\left(s a_{1}, a_{2}\right), s\left(a_{1} a_{2}\right)\right),
\end{aligned}
$$

hence for all $s \in S, a_{1}, a_{2} \in A$ it holds

(iv) $l\left(s, a_{1} a_{2}\right)=l\left(s, a_{1}\right) l\left(s a_{1}, a_{2}\right)$.

Since $R$ is a unital $A$-right system, from (iv') we get

( $\nabla) l\left(s, 1^{A}\right)=1^{B}$ for all $s \in S$.

We denote an admissible $(B, A)$-bisystem $B \times S$ by $A \stackrel{B, S}{\longrightarrow} B$. From Lemma 2.1 and $(\mathrm{i})-(\nabla)$ it follows:

LEMCMA 2.2. The monoid automata are in 1-1 correspondence with the admissible bisystems.

We define composition of admissible $(B, A)$-bisystem $B \times S$ and admissible $(C, B)$-bisystem $C \times S^{\prime}$ to be the admissible $(C, A)$-bisystem $C \times S^{\prime} \times S$ with right and left multiplication

$$
\left(c, s^{\prime}, s\right) a=\left(\left(c, s^{\prime}\right) l(s, a), s a\right), \quad c^{\prime}\left(c, s^{\prime}, s\right)=\left(c^{\prime} c, s^{\prime}, s\right) .
$$

LEMMA 2.3. With respect to the composition just defined the admissible bisystems in Set form a category Set-Abi $\rightarrow$ and the correspodence $\mathrm{G}$ between monoid automata and admissible bisystems described in Lemma 2.1, 2.2 is a functor

$$
G: \text { Set-Maut } \rightarrow \text { Set-Abi } \overrightarrow{ }
$$

We consider the behaviour of $G$ with respect to parallel connection (i.e. cartesian product). nection

Let $A \underset{l, m}{\stackrel{S}{\longrightarrow}} B, A^{\prime} \underset{l^{\prime}, m^{\prime}}{\stackrel{S^{\prime}}{\longrightarrow}} B^{\prime}$ be monoid automata with the parallel con-

$$
(A \underset{l, m}{\stackrel{S}{\longrightarrow}} B) \times\left(A^{\prime} \underset{l^{\prime}, m^{\prime}}{\stackrel{S^{\prime}}{\longrightarrow}} B^{\prime}\right)=A \times A^{\prime} \underset{l^{\prime \prime}, m^{\prime \prime}}{\stackrel{S \times S^{\prime}}{\longrightarrow}} B \times B^{\prime}
$$

and the corresponding admissible bisystem

$$
G\left(A \times A^{\prime} \stackrel{S \times S^{\prime}}{\longrightarrow} B \times B^{\prime \prime}\right)=A \times m^{\prime \prime} \stackrel{B \times B^{\prime}, S \times S^{\prime \prime}}{\longrightarrow} B \times B^{\prime}
$$

given by

$$
\begin{aligned}
\left(b, b^{\prime}, s, s^{\prime}\right)\left(a, a^{\prime}\right) & =\left(\left(b, b^{\prime}\right) l^{\prime \prime}\left(s, s^{\prime}, a, a^{\prime}\right),\left(s, s^{\prime}\right)\left(a, a^{\prime}\right)\right) \\
& =\left(b l(s, a), b^{\prime} l^{\prime}\left(s^{\prime}, a^{\prime}\right),\left(s a, s^{\prime} a^{\prime}\right)\right) .
\end{aligned}
$$

Thus if we define the cartesian product of admissible bisystems $A \stackrel{B, S}{\rightarrow} B, \ldots$ as the cartesian product in the sense of the theory of heterogeneous algebras, considering $A \stackrel{B, S}{\longrightarrow} B$ as a heterogeneous algebra with a triple $\langle A, B, S\rangle$ of carrier sets, we get:

LEMCMA 2.4. The functor $G$ is compatible with cartesian products.

We are mainly interested in sequential automata of the form $\times \boldsymbol{X}$ $\underset{l, m}{\stackrel{s}{\longrightarrow}} \times \boldsymbol{Y}$, where $\boldsymbol{X}=\left\langle X_{1}, \ldots, X_{n}\right\rangle, \boldsymbol{Y}=\left\langle Y_{1}, \ldots, Y_{k}\right\rangle$ are sequences of sets $\bar{X}_{i}, Y_{j}$ respectively. We call the triple

$$
\left\langle\boldsymbol{X}, \times \boldsymbol{X}_{l, m} \stackrel{s}{\longrightarrow} \times \boldsymbol{Y}, \boldsymbol{Y}\right\rangle
$$

a heterogeneous sequential automaton with an $n$-tuple $\boldsymbol{X}$ of input sets and a $k$-tuple $\boldsymbol{Y}$ of output sets. The h.s.a. (2.1) is considered as a morphism from $\boldsymbol{X}$ to $\boldsymbol{Y}$. If

$$
\left\langle\boldsymbol{X}^{\prime}, \times \boldsymbol{X}^{\prime} \underset{l^{\prime}, m^{\prime}}{\stackrel{S}{\longrightarrow}} \times \boldsymbol{Y}^{\prime}, \boldsymbol{Y}^{\prime}\right\rangle
$$

for $\boldsymbol{X}^{\prime}=\left\langle X_{1}^{\prime}, \ldots, X_{n^{\prime}}^{\prime}\right\rangle, \boldsymbol{Y}^{\prime}=\left\langle Y_{1}^{\prime}, \ldots, Y_{k^{\prime}}^{\prime}\right\rangle$ is a second h.s.a., then serial and parallel connection of (2.1) and (2.2) are again h.s.a. and respectively defined by

$$
\left\langle\boldsymbol{X},(\times \boldsymbol{X} \underset{l, m}{\stackrel{S}{\longrightarrow}} \times \boldsymbol{Y})\left(\times \boldsymbol{X}^{\prime} \underset{l^{\prime}, m^{\prime}}{\stackrel{S^{\prime}}{\longrightarrow}} \times \boldsymbol{Y}^{\prime}\right), \boldsymbol{Y}^{\prime}\right\rangle \quad \text { for } \quad \boldsymbol{Y}=\boldsymbol{X}^{\prime},
$$

The category of all h.s.a. in Set with serial connection (2.3) as composition is denoted by Set-Auth $\rightarrow$.

It arises the question to find an adequate passage from h.s.a. to admissible bisystems. A candidate for such a passage is the admissible 
bisystem

$$
G(\varepsilon(\boldsymbol{X}) F(\times \boldsymbol{X} \underset{l, m}{\stackrel{S}{\longrightarrow}} \times \boldsymbol{Y}) \mu(\boldsymbol{Y}))
$$

which is called the characteristic bisystem of the h.s.a. (2.1) and is denoted by $\operatorname{char}\langle\boldsymbol{X}, \times \boldsymbol{X} \underset{l, m}{\stackrel{S}{\longrightarrow}} \times \boldsymbol{Y}, \boldsymbol{Y}\rangle$.

LEMaA 2.5. As a heterogeneous algebra the characteristic bisystem of a h.s.a. is isomorphio to the characteristic bisystem in the sense of $\mathbb{E}$. S. Bainbridge [1] of the same h.s.a.

Proof is left to the reader.

Unfortunately it is hard to get an insight in preservation properties of the correspondence char.

Let us for instance consider parallel connection. It holds

$$
\mu\left(\left[\boldsymbol{Y}, \boldsymbol{Y}^{\prime}\right]\right)=\mu\left(\times \boldsymbol{Y}, \times \boldsymbol{Y}^{\prime}\right)\left(\mu(\boldsymbol{Y}) \times \mu\left(\boldsymbol{Y}^{\prime}\right)\right)
$$

and therefore by Lemma 1.2 and (2.5)

$$
\begin{aligned}
& \text { (2.6) } \quad \operatorname{char}\left(\langle\boldsymbol{X}, \times \boldsymbol{X} \underset{l, m}{\stackrel{S}{\longrightarrow}} \times \boldsymbol{Y}, \boldsymbol{Y}\rangle \times\left\langle\boldsymbol{X}^{\prime}, \times \boldsymbol{X}^{\prime} \underset{l^{\prime}, m^{\prime}}{S^{\prime}} \times \boldsymbol{Y}^{\prime}, \boldsymbol{Y}^{\prime}\right\rangle\right) \\
& =G\left(\varepsilon\left(\left[\boldsymbol{X}, \boldsymbol{X}^{\prime}\right]\right) F^{\prime}\left(\left(\times \boldsymbol{X}_{l, m}^{\stackrel{S}{\longrightarrow}} \times \boldsymbol{Y}\right) \times\left(\times \boldsymbol{X}^{\prime} \frac{S^{\prime}}{l^{\prime}, m^{\prime}} \times \boldsymbol{Y}^{\prime}\right)\right) \mu\left(\left[\boldsymbol{Y}, \boldsymbol{Y}^{\prime}\right]\right)\right) \\
& =G\left(\varepsilon\left(\left[\boldsymbol{X}, \boldsymbol{X}^{\prime}\right]\right) \mu\left(\times \boldsymbol{X}, \times \boldsymbol{X}^{\prime}\right)\left(F\left(\times \boldsymbol{X}_{l, m}^{S} \times \boldsymbol{Y}\right) \times F\left(\times \boldsymbol{X}^{\prime} \frac{S^{\prime}}{l^{\prime}, m^{\prime}} \times \boldsymbol{Y}\right)\right)(\mu(\boldsymbol{Y}) \times\right. \\
& \left.\times \mu\left(\boldsymbol{Y}^{\prime}\right)\right) \text {. }
\end{aligned}
$$

Since

$$
\begin{array}{r}
\varepsilon\left(\left[\boldsymbol{X}, \boldsymbol{X}^{\prime}\right]\right) \mu\left(\times \boldsymbol{X}, \times \boldsymbol{X}^{\prime}\right) \cong\left(\varepsilon(\boldsymbol{X}) \times \varepsilon\left(\boldsymbol{X}^{\prime}\right)\right) \varepsilon\left(\times \boldsymbol{X}, \times \boldsymbol{X}^{\prime}\right) \mu\left(\times \boldsymbol{X}, \times \boldsymbol{X}^{\prime}\right) \\
\underset{\neq}{\neq} \varepsilon(\boldsymbol{X}) \times \varepsilon(\boldsymbol{X})
\end{array}
$$

we are not able to reduce line $3 / 4$ of (2.6) to an. expression which (at least up to isomorphy) may be written only in terms of char. Also the fact that (replacing $\times \boldsymbol{X}, \times \boldsymbol{X}^{\prime}$ by $\left.X, X^{\prime}\right)$

$$
\left\langle l_{1}\left(\left(1,1^{\prime}\right),-\right), l_{1}\left(\left(1,1^{\prime}\right),-1\right\rangle, \quad\left(1,1^{\prime}\right): \mathbf{1} \rightarrow \varepsilon\left(X, X^{\prime}\right) \mu\left(X, X^{\prime}\right),\right.
$$

where $1=1^{X^{*}}, 1^{\prime}=1^{X^{*}} ; \mathbf{1}=X^{*} \times{X^{\prime *}}^{1} \frac{I}{1_{X^{*} \times X^{*}, t_{X^{*} \times X^{*}}}} X^{*} \times X^{\prime *}$ being an identity monoid automaton; is a (sequential) homomorphism opens no way since it is no homomorphism of monoid automata.

\section{Construction of general coding maps $\varepsilon, \mu, v$}

We assume the existence of three kinds of maps $\varepsilon, \mu, \nu$, which are defined on all sequences $\boldsymbol{A}=\left\langle A_{1}, \ldots, A_{n}\right\rangle, n=1,2, \ldots$, of monoids $A_{i}$ and
satisfy the following set of conditions: $\varepsilon 1 . \quad \varepsilon(\boldsymbol{A})=\times \boldsymbol{A} \underset{l_{\boldsymbol{A}}, m_{\boldsymbol{A}}}{\stackrel{s_{\boldsymbol{A}}}{\longrightarrow}} E_{\boldsymbol{A}}$ is a monoid automaton.

$\varepsilon 2 . \quad \varepsilon(\boldsymbol{A})=A \frac{I}{1_{A}, t_{\mathcal{A}}} A$ if $\boldsymbol{A}=\langle A\rangle$.

83. $\varepsilon\left(\boldsymbol{X}^{*}\right)=\varepsilon(\boldsymbol{X})$.

E1. $E_{\boldsymbol{A}}$ is a monoid.

E2. $E_{A}=A$ if $\boldsymbol{A}=\langle A\rangle$.

E3. $E_{X^{*}}=(\times X)^{*}$.

11. $\mu(\boldsymbol{A})=M_{\boldsymbol{A}} \frac{I}{\mu_{\boldsymbol{A}}, t_{M_{\boldsymbol{A}}}} \times \boldsymbol{A}$ is a monoid automaton.

$\mu 2$. $\mu(\boldsymbol{A})=A \underset{1_{\boldsymbol{A}}, t_{\boldsymbol{A}}}{\longrightarrow} A$ if $\boldsymbol{A}=\langle A\rangle$.

⒊ $\mu\left(\boldsymbol{X}^{*}\right)=\mu(\boldsymbol{X})$.

II1. $M_{\boldsymbol{A}}$ is a monoid.

M2. $M_{A}=A$ if $\boldsymbol{A}=\langle A\rangle$.

M3. $M_{\boldsymbol{X}^{*}}=(\times \boldsymbol{X})^{*}$.

v1. $v(\boldsymbol{A})=E_{\boldsymbol{A}} \underset{{ }_{\boldsymbol{v}_{\boldsymbol{A}}, t_{E_{A}}}}{\mathrm{I}}(\times \boldsymbol{A}) / \lambda_{\boldsymbol{A}}$ is a monoid automaton.

v2. $v(\boldsymbol{A})=A \underset{1}{\stackrel{1}{1_{A}, t_{A}}} A$ if $\boldsymbol{A}=\langle A\rangle$.

v3. $v\left(\boldsymbol{X}^{*}\right)=\mu(\boldsymbol{X})$.

Here $\varepsilon(\boldsymbol{X}), \mu(\boldsymbol{X})$ are the monoid automata already defined in Section 1. $\lambda_{\boldsymbol{A}}$ is a congruence in $\times \boldsymbol{A} ;(\times \boldsymbol{A}) / \lambda_{\boldsymbol{A}}$ is the factor monoid of $\times \boldsymbol{A}$ with respect to $\lambda_{A}$.

Obviously E1, E2, E3, and M1, M2, M3, are consequences of $\varepsilon 1, \varepsilon 2, \varepsilon 3$, and $\mu 1, \mu 2, \mu 3$, respectively.

Such maps $\varepsilon, \mu, \nu$ are assumed to be realized in any way. We construct one realization for them:

We assume every monoid $A$ to be represented in the form

$$
A=\varphi\left(X^{*} / x\right) ; \quad x=0 \text { and } \varphi=1_{X^{*}} \quad \text { if } \quad A=X^{*},
$$

where $X$ is a set, $x$ is a suitable congruence in the free monoid $X^{*}$, and $\varphi$ is a suitable isomorphism of monoids. This representation is fixed.

Let

$$
\boldsymbol{A}=\left\langle A_{1}, \ldots, A_{n}\right\rangle=\left\langle\varphi_{1}\left(X_{1}^{*} \mid x_{1}\right), \ldots, \varphi_{n}\left(X_{n}^{*} \mid x_{n}\right)\right\rangle
$$

be a sequence of monoids and write

$$
\boldsymbol{\kappa}=\left\langle x_{1}, \ldots, x_{n}\right\rangle, \quad \boldsymbol{\varphi}=\left\langle\varphi_{1}, \ldots, \varphi_{n}\right\rangle .
$$

Then the product $\times \boldsymbol{k}=\varkappa_{1} \times \ldots \times \varkappa_{n}$ is a congruence in $\times \boldsymbol{X}^{*} .(\times \boldsymbol{X})^{*} /(\times \boldsymbol{\kappa})$ denotes the image of the monoid $(X \boldsymbol{X})^{*}$ under the canonical homomorphism $\times \boldsymbol{X}^{*} \rightarrow\left(X \boldsymbol{X}^{*}\right) /(\times \boldsymbol{k})$ of $\times \boldsymbol{X}^{*}$ onto the factor monoid $\left(\times \boldsymbol{X}^{*}\right) /(\times \boldsymbol{k})$ of $\times \boldsymbol{X}^{*}$ 
with respect to $\times \boldsymbol{\kappa}$.

We get

$$
(\times \varphi)\left((\times \boldsymbol{X})^{*} /(\times \boldsymbol{k})\right) \subseteq(\times \varphi)\left(\left(\boldsymbol{X}^{*}\right) /(\times \boldsymbol{k})\right)=\times \boldsymbol{A}
$$

we define

$$
M_{\boldsymbol{A}}=(\times \varphi)\left((\times \boldsymbol{X})^{*} /(\times \boldsymbol{\kappa})\right), \quad \mu_{\boldsymbol{A}}=M_{\boldsymbol{A}} \stackrel{\varrho}{\rightarrow} \times \boldsymbol{A} .
$$

Then $\mu 1, \mu 2, \mu 3$ are satisfied.

We introduce the general notions:

$$
\begin{gathered}
\tilde{A}_{1}=A_{1} \times \ldots \times A_{i-1} \times\left\{1^{A_{i}}\right\} \times A_{i+1} \times \ldots \times A_{n}, \\
\mathcal{S}^{\boldsymbol{A}}=\bigcup_{i=1}^{n} \tilde{A}_{i}, \quad \boldsymbol{X}^{*} / \kappa=\left\langle X_{1}^{*}\left|x_{1}, \ldots, X_{n}^{*}\right| x_{n}\right\rangle .
\end{gathered}
$$

If $S^{X^{*}} /(\times \boldsymbol{k})$ denotes the set of all equivalence classes $\bmod \times \boldsymbol{k}$ of the elements of $S^{X^{*}}$ we have

$$
S^{X^{*} / \boldsymbol{r}}=S^{X^{*}} /(X \boldsymbol{x}) \subseteq\left(\times \boldsymbol{X}^{*}\right) /(\times \boldsymbol{\kappa}) .
$$

For $w=\left\langle w_{1}, \ldots, w_{n}\right\rangle \in \times \boldsymbol{X}^{*}$ the congruence class mod $\times \boldsymbol{\kappa}$ containing $w$ is denoted by $\bar{w}$. Obviously $\bar{w}=\left\langle\bar{w}_{1}, \ldots, \bar{w}_{n}\right\rangle$, where $\tilde{w}_{i}$ for $w_{i} \in X_{i}$ denotes the congruence class $\bmod \kappa_{i}$ containing $w_{i}$. If $u \in S^{X^{*}}$, then as already mentioned in Section 1 there are uniquely determined elements $v \in(\times \boldsymbol{X})^{*}$, $u^{\prime} \in S^{X^{*}}$ such that $u w=v u^{\prime}$. Hence $\bar{u} \bar{w}=\bar{v} \bar{u}^{\prime}$. In $S^{X^{*} / k}$ we define the equivalence $q$ generated by the pairs $\left(\bar{u}, \bar{u}_{0}\right)$ such that

and there are

$$
\bar{u}, \bar{u}_{0} \in S^{\mathbf{X}^{*} / k} \quad\left(u, u_{0} \in \boldsymbol{S}^{\mathbf{X}^{*}}\right)
$$

for which

$$
\bar{v}, \bar{v}_{0} \in(\times \boldsymbol{X})^{*} /(\times \boldsymbol{\kappa}) \quad\left(v, v_{0} \in(\times \boldsymbol{X})^{*}\right)
$$

$$
\bar{v} \bar{u}=\bar{v}_{0} \bar{u}_{0} .
$$

We define $S_{\boldsymbol{A}}$ as the factor set $S_{\boldsymbol{A}}=S^{x^{*} / \boldsymbol{\kappa}} / q$, and the map

$$
m_{\boldsymbol{A}}: S_{\boldsymbol{A}} \times(\times \boldsymbol{A}) \rightarrow S_{\boldsymbol{A}}, \quad m_{\boldsymbol{A}}(\overline{\bar{u}},(\times \varphi)(\bar{w}))=u^{\prime}
$$

if exist $\bar{u}^{\prime} \in S^{\boldsymbol{X}^{*} / \kappa}$ and $\bar{v} \in(\times \boldsymbol{X})^{*} /(\times \boldsymbol{\kappa})$ such that $\bar{u} \bar{w}=\bar{v} \bar{u}^{\prime}$.

Here $\overline{\bar{u}}$ denotes the equivalence class $\bmod q$ containing the element $\bar{u}$ $\in S^{X^{*} / \boldsymbol{x}}$, and so on.

It is clear that $m_{A}$ is defined everywhere. The uniqueness can be proved as follows:

Let be $u, u^{\prime}, u_{0}, u_{0}^{\prime} \in S^{\boldsymbol{X}^{*}}, v, v_{0} \in(\times \boldsymbol{X})^{*}$,

$$
\bar{u} \bar{w}=\bar{v} \bar{u}^{\prime}, \quad \bar{u}_{0} \bar{w}=\bar{v}_{0} \bar{u}_{0}^{\prime}, \quad \text { and } \quad \overline{\bar{u}}=\overline{\bar{u}}_{0} .
$$

Because of $\overline{\bar{u}}=\overline{\bar{u}}_{0}$ there are $u^{(i)}, u_{0}^{(i)} \in S^{X^{*}}, v^{(i)}, v_{0}^{(i)} \in(X \boldsymbol{X})^{*}$ such that

$$
\begin{gathered}
\bar{u}^{(1)}=u, \quad u_{0}^{(k)}=u_{0}, \quad u_{0}^{(i)}=u^{(i+1)}, \quad i=1, \ldots, k-1, \\
\overline{v^{(i)}} \overline{u^{(i)}}=\overline{v_{0}^{(i)}} \overline{u_{0}^{(i)}}, \quad i=1, \ldots, k .
\end{gathered}
$$

Compute $u_{i} \in S^{\mathbf{X}^{*}}, v_{i} \in(\times \boldsymbol{X})^{*}$ according to

Then it holds

$$
u^{(i)} w: v_{i} u_{i}, \quad i=2, \ldots, k-1 \text {. }
$$

$$
\begin{aligned}
& \overline{v^{(1)} v} \bar{u}^{\prime}=\overline{v^{(1)}} \bar{v} \bar{u}^{\prime}=\overline{v^{(1)}} \bar{u} \bar{w}=\overline{v^{(1)}} \overline{u^{(1)}} \bar{w} \\
& =\overline{v_{0}^{(1)}} \overline{u_{0}^{(1)}} \bar{w}=\overline{v_{0}^{(1)}} \overline{u^{(2)}} \bar{w}=\overline{v_{0}^{(1)}} \bar{v}_{2} \bar{u}_{2}=\overline{v_{0}^{(1)} v_{2}} \bar{u}_{2}, \\
& \overline{v^{(2)}} \bar{v}_{2} \bar{u}_{2}=\overline{v^{(2)}} \overline{u^{(2)}} \bar{w}=\overline{v_{0}^{(2)}} \overline{u_{0}^{(2)}} \bar{w}=\overline{v_{0}^{(2)}} \overline{u^{(3)}} \bar{w}=\overline{v_{0}^{(2)}} \bar{v}_{3} \bar{u}_{3} \text {, } \\
& \overline{v^{(3)}} \bar{v}_{3} \bar{u}_{3}=\overline{v^{(3)}} \overline{u^{(3)}} \bar{w}=\overline{v_{0}^{(3)}} \overline{u_{0}^{(3)}} \bar{w}=\overline{v_{0}^{(3)}} \overline{u^{(4)}} \bar{w}=\overline{v^{(3)}} \bar{v}_{4} \bar{u}_{4} \\
& \ldots \ldots \ldots \ldots \ldots \ldots \ldots \ldots \ldots \ldots \ldots \ldots \\
& \overline{v^{(k-1)}} \bar{v}_{k-1} \bar{u}_{k-1}=\overline{v^{(k-1)}} \overline{u^{(k-1)}} \bar{w}=\overline{v_{0}^{(k)}} \overline{u_{0}^{(k)}} \bar{w}=\overline{v_{0}^{(k)}} \bar{u}_{0} \bar{w}=\overline{v_{0}^{(k)}} \bar{v}_{0} \bar{u}_{0}^{\prime} .
\end{aligned}
$$

Hence

$$
\overline{\overline{u^{\prime}}}=\overline{\bar{u}}_{2}=\overline{\bar{u}}_{3}=\ldots=\overline{\bar{u}}_{k-1}=\overline{\overline{u_{0}^{\prime}}} \text {. }
$$

$\left\langle S_{\boldsymbol{A}}, m_{\boldsymbol{A}}\right\rangle$ is a unital $\times \boldsymbol{A}$-system. Indeed, let be

$$
\begin{gathered}
m_{\boldsymbol{A}}(\overline{\bar{u}},(\times \varphi)(\bar{w}))=\overline{\overline{u^{\prime}}}, \quad \bar{u} \bar{w}=\bar{v} \bar{u}^{\prime}, \\
m_{\boldsymbol{A}}\left(\overline{\bar{u}}^{\prime},(\times \varphi) \overline{\left.\left(w^{\prime}\right)\right)}=\overline{\overline{u^{\prime \prime}}}, \overline{u^{\prime}} \overline{w^{\prime}}=\overline{v^{\prime}} \overline{u^{\prime \prime}},\right. \\
m_{\boldsymbol{A}}\left(\overline{\bar{u}},(\times \varphi)\left(\bar{w} \overline{w^{\prime}}\right)\right)=\overline{\overline{u^{\prime \prime \prime}}}, \quad \bar{u} \bar{w} \overline{w^{\prime}}=\overline{v^{\prime \prime}} \overline{u^{\prime \prime \prime}} .
\end{gathered}
$$

We claim that $\overline{\overline{u^{\prime \prime}}}=\overline{\overline{u^{\prime \prime \prime}}}$. This is a consequence of

$$
\overline{v^{\prime \prime}} \overline{u^{\prime \prime \prime}}=\bar{u} \overline{w w^{\prime}}=\bar{v} \bar{u}^{\prime} \bar{w}^{\prime}=\bar{v} \bar{v} \overline{u^{\prime \prime}}=\overline{v v^{\prime}} \overline{u^{\prime \prime}} \text {. }
$$

Consider the action of the identity $1^{\times \boldsymbol{A}}=(\times \varphi)\left(1^{\left(\times \boldsymbol{X}^{*}\right) /(\times x)}\right)$ on an element $\overline{\bar{u}} \in S_{\boldsymbol{A}}:$ It holds

$$
\bar{u} 1^{\left(\times \boldsymbol{X}^{*}\right) /(\times \kappa)}=\bar{u}=1^{\left(\times \boldsymbol{X}^{*}\right) /(\times \kappa)} \bar{u}
$$

and $1^{\left(\times X^{*}\right) /(\times \kappa)} \in(\times \boldsymbol{X})^{*} /(\times \boldsymbol{\kappa})$, hence $m_{\boldsymbol{A}}\left(\overline{\bar{u}}, 1^{\times \boldsymbol{A}}\right)=\overline{\bar{u}}$.

We describe the construction of $E_{\boldsymbol{A}}$. Motivated by the expressions

$$
m_{\boldsymbol{A}}(\overline{\bar{u}},(\times \varphi)(\bar{w}))=\overline{\bar{u}}^{\prime}, \quad \bar{u} \bar{w}=\bar{v} \bar{u}^{\prime},
$$

we consider the congruence relation $r$ on $(\times \boldsymbol{X})^{*} /(\times \boldsymbol{\kappa})$ generated by the pairs

$$
\left\langle\bar{v}, \overline{\left.v^{\prime}\right\rangle} \quad \text { with } \quad \bar{v}_{0} \bar{v} \overline{u^{\prime}}=\overline{v_{0}^{\prime} v^{\prime}} \overline{u^{\prime \prime}} \quad \text { and } \quad \bar{v}_{0} \overline{u_{0}^{\prime}}=\overline{v_{0}^{\prime}} \overline{v_{0}^{\prime \prime}}\right.
$$

for suitable $u^{\prime}, u_{0}^{\prime}, u^{\prime \prime}, u_{0}^{\prime \prime} \in S^{\boldsymbol{X}^{*}}, v_{0}, v_{0}^{\prime} \in(\times \boldsymbol{X})^{*}$. 
Take

$$
E_{\boldsymbol{A}}=(\times \varphi)\left((\times \boldsymbol{X})^{*} /(\times \boldsymbol{x})\right) /((\times \varphi) \times(\times \varphi)) r=\chi\left(\left((\times \boldsymbol{X})^{*} /(\times \boldsymbol{x})\right) / r\right),
$$

where $\chi:\left((\times \boldsymbol{X})^{*} /(\times \boldsymbol{x})\right) / r \rightarrow E_{\boldsymbol{A}}$ is a suitable isomorphism such that the following diagram is commutative $\left(l_{\Phi}\right.$ a map induced as a restriction of $\operatorname{map} \Phi)$ :

We define

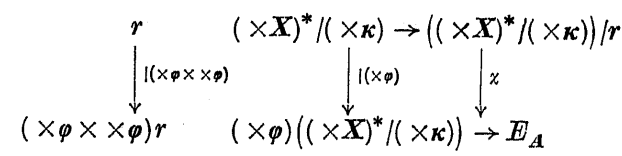

$$
l_{\boldsymbol{A}}: S_{\boldsymbol{A}} \times(\times \boldsymbol{A}) \rightarrow E_{\boldsymbol{A}}, \quad l_{\boldsymbol{A}}(\overline{\bar{u}},(\times \varphi)(\bar{w}))=\chi_{\bar{v}},
$$

if exist $\overline{u^{\prime}} \in \mathbb{S}^{\boldsymbol{X}^{*} / \kappa}$ and $\bar{v} \in(\times \boldsymbol{X})^{*} /(\times \boldsymbol{k})$ such that $\bar{u} \bar{w}=\bar{v} \overline{u^{\prime}}$.

Here $\overline{\bar{v}}$ denotes the congruence class $\bmod r$ containing the element $\bar{v} \in(X \boldsymbol{X})^{*} /(\times \boldsymbol{\kappa})$, and so on.

It is clear that $l_{\boldsymbol{A}}$ is everywhere defined. We show that $l_{\boldsymbol{A}}$ is uniquely defined. Indeed, let

$$
\begin{array}{cc}
l_{\boldsymbol{A}}(\overline{\bar{u}},(\times \varphi)(\bar{w}))=\chi \overline{\bar{v}}, & \bar{u} \bar{w}=\bar{v} \overline{u^{\prime}} ; \\
l_{\boldsymbol{A}}\left(\overline{\bar{u}}_{0},(\times \varphi)(\bar{w})\right)=\chi \overline{\bar{v}}_{0}, & \bar{u}_{0} \bar{w}=\bar{v}_{0} \overline{\bar{u}_{0}^{\prime}},
\end{array}
$$
and $\overline{\bar{u}}=\overline{\bar{u}}_{0}$. Because of $\overline{\bar{u}}=\overline{\bar{u}}_{0}$ there are $u^{(i)}, u_{0}^{(i)} \in \mathcal{S}^{X^{*}}, v^{(i)}, v_{0}^{i} \in(\times \boldsymbol{X})^{*}$
such that

$$
\begin{array}{ll}
u^{(1)}=u, \quad u_{0}^{(k)}=u_{0}, \quad u_{0}^{(i)}=u^{(i+1)}, \quad i=2, \ldots, k-1, \\
\overline{v^{(i)}} \overline{u^{(i)}}=\overline{v_{0}^{(i)}} \overline{u_{0}^{(i)}}, \quad i=1, \ldots, k .
\end{array}
$$

Compute $u_{i} \in S^{\boldsymbol{X}^{*}}, v_{i} \in(\times \boldsymbol{X})^{*}$ according to

$$
u^{(i)} w=v_{i} u_{i}, \quad i=2, \ldots, l_{i}-1 .
$$

The same formulas as above yield

$$
\overline{\bar{v}}=\overline{\bar{v}}_{2}=\overline{\bar{v}}_{3}=\ldots=\overline{\bar{v}}_{k-1}=\overline{\bar{v}}_{0} .
$$

In order to prove that $\varepsilon(\boldsymbol{A})$ is a monoid automaton we have to show the validity of the axioms

$$
\begin{gathered}
l_{\boldsymbol{A}}(\overline{\bar{u}},(\times \varphi)(\bar{w})) l_{\boldsymbol{A}}\left(m_{\boldsymbol{A}}(\overline{\bar{u}},(\times \varphi)(\bar{w})),(\times \varphi)\left(\overline{w^{\prime}}\right)\right)=l_{\boldsymbol{A}}\left(\overline{\bar{u}},(\times \varphi)\left(\bar{w} \overline{w^{\prime}}\right)\right), \\
l_{\boldsymbol{A}}\left(\overline{\bar{u}}, 1^{\times \boldsymbol{A}}\right)=1^{E_{\boldsymbol{A}}} .
\end{gathered}
$$

The second of these axioms follows from

$$
\bar{u} 1^{\left(\times X^{*}\right) /(\times \kappa)}=\bar{u}=1^{(\times X) /(\times x)} \bar{u} .
$$

We prove the first of the axioms mentioned above. Let be

$$
\begin{gathered}
m_{\boldsymbol{A}}(\overline{\bar{u}},(\times \varphi)(\bar{w}))=\overline{\overline{u^{\prime}}}, \\
l_{\boldsymbol{A}}(\overline{\bar{u}},(\times \varphi)(\bar{w}))=\overline{\bar{v}}, \quad \overline{\bar{u}} \bar{w}=\overline{\bar{v} \overline{u^{\prime}},} \\
\left.l_{\boldsymbol{A}}\left(\overline{\overline{u^{\prime}}},(\times \varphi) \overline{\left(\bar{w}^{\prime}\right.}\right)\right)=\chi \overline{\overline{v^{\prime}}}, \quad \overline{\bar{u}^{\prime}} \overline{\overline{w^{\prime}}}=\overline{v^{\prime}} \overline{u^{\prime \prime}}, \\
l_{\boldsymbol{A}}\left(\overline{\bar{u}},(\times \varphi) \overline{\left(w v^{\prime}\right)}\right)=\overline{\overline{v^{\prime \prime}}}, \quad \overline{\bar{u} \overline{w w^{\prime}}}=\overline{v^{\prime \prime}} \overline{u^{\prime \prime \prime}} .
\end{gathered}
$$

The validity of the equation $\overline{\bar{v}} \overline{\bar{v}^{\prime}}=\overline{\overline{v^{\prime \prime}}}$ follows from

$$
\overline{\overline{v^{\prime \prime}}} \overline{\overline{u^{\prime \prime \prime}}}=\bar{u} \bar{w} \overline{w^{\prime}}=\bar{v} \overline{u^{\prime}} \overline{w^{\prime}}=\bar{v} \overline{v^{\prime}} \overline{u^{\prime \prime}} \text {. }
$$

We consider the congruence in $\left(X X^{*}\right) /(X \boldsymbol{x})$ generated by $r$ and denote it by the same letter $r$. Then we have a natural homomorphism

$$
v:\left((\times \boldsymbol{X})^{*} /(\times \boldsymbol{k})\right) / r \rightarrow\left(\left(\times \boldsymbol{X}^{*}\right) /(\times \boldsymbol{k})\right) / r
$$

which induces a homomorphism

$$
v_{A}: E_{A} \rightarrow(\times A) / \lambda_{A}, \quad \lambda_{A}=((\times \varphi) \times(\times \varphi)) r,
$$

where one has to take into consideration, that

$$
\begin{aligned}
& E_{\boldsymbol{A}}=(\times \varphi)\left((\times \boldsymbol{X})^{*} /(\times \boldsymbol{\kappa})\right) /((\times \varphi) \times(\times \varphi)) r, \\
& \times \boldsymbol{A}=(\times \varphi)\left(\left(\times \boldsymbol{X}^{*}\right) /(\times \boldsymbol{\kappa})\right) .
\end{aligned}
$$

We define

$$
v(\boldsymbol{A})=E_{\boldsymbol{A}} \underset{{ }_{{ }_{\boldsymbol{A}}, t_{E_{A}}}}{\stackrel{I}{\longrightarrow}}(\times \boldsymbol{A}) / \lambda_{\boldsymbol{A}} .
$$

LEMMA 3.1. The maps $\varepsilon, \mu, v$, constructed above satisfy conditions $\varepsilon 1, \varepsilon 2, \varepsilon 3, \mu 1, \mu 2, \mu 3, \nu 1, \nu 2, \nu 3$.

\section{Heterogeneous monoid automata}

By means of the auxiliary maps $\varepsilon, \mu, \nu$, introduced in Section 3 we are able to give a general definition of a heterogeneous monoid automaton (h.m.a.):

A h.m.a. is a sequence of the form

$$
(S)=\langle[\underline{\boldsymbol{A}}], \underline{\boldsymbol{A}}, \underline{\boldsymbol{R}}, \times \boldsymbol{R} \underset{l, m}{\stackrel{S}{\longrightarrow}} \times \boldsymbol{B}, \underline{\boldsymbol{B}},[\underline{\boldsymbol{B}}]\rangle:[\underline{\boldsymbol{A}}] \rightarrow[\underline{\boldsymbol{B}}],
$$


where

$$
\begin{gathered}
\underline{\boldsymbol{A}}=\left\langle\boldsymbol{A}_{1}, \ldots, \boldsymbol{A}_{n}\right\rangle, \quad \underline{\boldsymbol{R}}=\left\langle\boldsymbol{R}_{1}, \ldots, \boldsymbol{R}_{n}\right\rangle, \\
\boldsymbol{R}=\left\langle R_{1}, \ldots, R_{n}\right\rangle, \quad R_{i}=\left(\times \boldsymbol{R}_{i}\right) / \lambda_{\boldsymbol{R}_{i}}, \quad \boldsymbol{E}_{\boldsymbol{A}_{i}}=E_{\boldsymbol{R}_{i}}, \\
\underline{\boldsymbol{B}}=\left\langle\boldsymbol{B}_{1}, \ldots, \boldsymbol{B}_{k}\right\rangle, \quad \boldsymbol{B}=\left\langle B_{1}, \ldots, B_{k}\right\rangle, \quad B_{j}=M_{\boldsymbol{B}_{j}}, \\
j=1, \ldots, k,
\end{gathered}
$$

and where $\times \boldsymbol{R} \underset{l, m}{\stackrel{S}{\longrightarrow}} \times \boldsymbol{B}$ is an ordinary (= homogeneous) monoid automaton.

T.wo h.m.a (S') (cf. (1)) and ( $\left.S^{\prime}\right)$ (cf. (2) below):

(2) $\left(S^{\prime}\right)=\left\langle\left[\underline{\boldsymbol{A}^{\prime}}\right], \underline{\boldsymbol{A}^{\prime}}, \underline{\boldsymbol{R}^{\prime}}, \times \boldsymbol{R}^{\prime} \frac{S^{\prime}}{l^{\prime}, m^{\prime}} \times \boldsymbol{B}^{\prime}, \underline{\boldsymbol{B}}^{\prime},\left[\underline{\boldsymbol{B}^{\prime}}\right]\right\rangle:\left[\underline{\boldsymbol{A}^{\prime}}\right] \rightarrow\left[\underline{\boldsymbol{B}}^{\prime}\right]$, where

$$
\begin{gathered}
\underline{\boldsymbol{A}}^{\prime}=\left\langle\boldsymbol{A}_{1}^{\prime}, \ldots, \boldsymbol{A}_{n^{\prime}}^{\prime}\right\rangle, \quad \underline{\boldsymbol{R}^{\prime}=\left\langle\boldsymbol{R}_{1}^{\prime}, \ldots, \boldsymbol{R}_{n^{\prime}}^{\prime}\right\rangle,} \\
\boldsymbol{R}^{\prime}=\left\langle R_{1}^{\prime}, \ldots, R_{n^{\prime}}^{\prime}\right\rangle, \quad R_{i}^{\prime}=\left(\times \boldsymbol{R}_{i}^{\prime}\right) / \lambda_{\boldsymbol{R}_{i}^{\prime}}, \quad E_{\boldsymbol{A}_{i}^{\prime}}=E_{\boldsymbol{R}_{i}^{\prime}}, \\
\underline{\boldsymbol{B}}^{\prime}=\left\langle\boldsymbol{B}_{1}^{\prime}, \ldots, \boldsymbol{B}_{k^{\prime}}^{\prime}\right\rangle, \quad \boldsymbol{B}^{\prime}=\left\langle B_{1}^{\prime}, \ldots, B_{k^{\prime}}^{\prime}\right\rangle, \quad B_{j}^{\prime}=M_{\boldsymbol{B}_{j}^{\prime}}, \\
j=1, \ldots, k^{\prime},
\end{gathered}
$$

are said to be equal, if

$$
[\underline{\boldsymbol{A}}]=\left[\underline{\boldsymbol{A}^{\prime}}\right], \quad[\underline{\boldsymbol{B}}]=\left[\underline{\boldsymbol{B}}^{\prime}\right]
$$

and

(4) $\quad\left(\varepsilon\left(\boldsymbol{A}_{1}\right) v\left(\boldsymbol{R}_{1}\right) \times \ldots \times \varepsilon\left(\boldsymbol{A}_{n}\right) v\left(\boldsymbol{R}_{n}\right)\right)$

$$
\begin{aligned}
\cdot(\times \boldsymbol{R} \underset{l, m}{\stackrel{S}{\longrightarrow}} \times \boldsymbol{B})\left(\mu\left(\boldsymbol{B}_{1}\right) \times \ldots \times \mu\left(\boldsymbol{B}_{k}\right)\right) \\
=\left(\varepsilon\left(\boldsymbol{A}_{1}^{\prime}\right) v\left(\boldsymbol{R}_{1}^{\prime}\right) \times \ldots \times \varepsilon\left(\boldsymbol{A}_{n^{\prime}}^{\prime}\right) v\left(\boldsymbol{R}_{n^{\prime}}^{\prime}\right)\right) \cdot \\
\cdot\left(\times \boldsymbol{R}^{\prime} \frac{s^{\prime}}{l^{\prime}, m^{\prime}} \times \boldsymbol{B}^{\prime}\right)\left(\mu\left(\boldsymbol{B}_{1}^{\prime}\right) \times \ldots \times \mu\left(\boldsymbol{B}_{k^{\prime}}^{\prime}\right)\right) .
\end{aligned}
$$

The composition of two h.m.a. (S), ( $\left.S^{\prime}\right)$ (cf. (1), (2)) is defined if

$$
[\underline{B}]=\left[\underline{A}^{\prime}\right] .
$$

The compositum $(S)\left(S^{\prime}\right)$ of $\left(S^{\prime}\right)$ and $\left(S^{\prime}\right)$ is again a h.m.a. which is computed in the following way. By definition, $\left(S^{\prime}\right)$ and $\left(S^{\prime}\right)$ are respectively equal to

(5) $\left\langle[\underline{\boldsymbol{A}}], \underline{\boldsymbol{A}}, \underline{\boldsymbol{R}},(\times \boldsymbol{R} \underset{l, m}{\stackrel{\boldsymbol{S}}{\longrightarrow}} \times \boldsymbol{B})\left(\mu\left(\boldsymbol{B}_{1}\right) \times \ldots \times \mu\left(\boldsymbol{B}_{k}\right)\right),[\underline{\boldsymbol{B}}],[\underline{\boldsymbol{B}}]\right\rangle$,

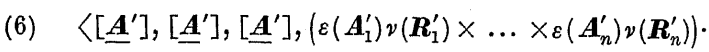

$$
\left.\cdot\left(\times \boldsymbol{R}^{\prime} \underset{l^{\prime} \cdot m^{\prime}}{\stackrel{S^{\prime}}{\longrightarrow}} \times \boldsymbol{B}^{\prime}\right), \underline{\boldsymbol{B}}^{\prime},\left[\underline{\boldsymbol{B}}^{\prime}\right]\right\rangle \text {. }
$$

In (6) [ $\left.\underline{A}^{\prime}\right]$ at the second and third place is considered as a sequence (of the same length as $\left[\underline{A}^{\prime}\right]$ at the first place, but) of sequences of length one (rather then of elements). The compositum $\left(S^{\prime}\right)\left(S^{\prime}\right)$ is given by

$$
\begin{aligned}
(7) \quad(S)\left(S^{\prime}\right)=\langle[\underline{\boldsymbol{A}}], \underline{\boldsymbol{A}}, \underline{\boldsymbol{R}},(\times \boldsymbol{R} \underset{l, m}{\stackrel{S}{\longrightarrow}} \times \boldsymbol{B}) \cdot \\
\cdot\left(\mu\left(\boldsymbol{B}_{1}\right) \times \ldots \times \mu\left(\boldsymbol{B}_{k}\right)\right)\left(\varepsilon\left(\boldsymbol{A}_{1}^{\prime}\right) v\left(\boldsymbol{R}_{1}^{\prime}\right) \times \ldots \times \varepsilon\left(\boldsymbol{A}_{n^{\prime}}^{\prime}\right) v\left(\boldsymbol{R}_{n^{\prime}}^{\prime}\right)\right) . \\
\left.\cdot\left(\times \boldsymbol{A}^{\prime} \frac{S^{\prime}}{l^{\prime}, m^{\prime}} \times \boldsymbol{B}^{\prime}\right), \underline{\boldsymbol{B}},\left[\underline{\boldsymbol{B}^{\prime}}\right]\right\rangle:[\underline{\boldsymbol{A}}] \rightarrow\left[\underline{\left.\boldsymbol{B}^{\prime}\right] .}\right.
\end{aligned}
$$

As easily can be seen the composition of h.m.a. is compatible with the equality of h.m.a. as parallel connection ((8)) does.

LEMMA 4.1. With the composition defined above the h.m.a. form a category Set-Mauth $\rightarrow$ in which parallel connection of two h.m.a. (S) and $\left(S^{\prime}\right)$ (cf. (1), (2)) is again a h.m.a. which is defined by

(8) $(S) \times\left(S^{\prime}\right)=\left\langle\left[\left[\underline{\boldsymbol{A}}, \underline{\boldsymbol{A}}^{\prime}\right]\right],\left[\underline{\boldsymbol{A}}, \underline{\boldsymbol{A}}^{\prime}\right],\left[\underline{\boldsymbol{R}}, \underline{\boldsymbol{R}}^{\prime}\right],\left(\times \boldsymbol{R} \frac{\boldsymbol{S}}{\overrightarrow{l, m}} \times \boldsymbol{B}\right) \times\right.$

$$
\begin{aligned}
\left.\times\left(\times \boldsymbol{R}^{\prime} \frac{s^{\prime}}{l^{\prime}, m^{\prime}}, \times \boldsymbol{B}^{\prime}\right),\left[\underline{\boldsymbol{B}}, \underline{\boldsymbol{B}^{\prime}}\right],\left[\left[\underline{\boldsymbol{B}}, \underline{\boldsymbol{B}^{\prime}}\right]\right]\right\rangle: \\
{[[\underline{\boldsymbol{A}}, \underline{\boldsymbol{A}}]] \rightarrow\left[\left[\underline{\boldsymbol{B}}, \underline{\boldsymbol{B}^{\prime}}\right]\right], }
\end{aligned}
$$

where

$$
\begin{gathered}
{\left[\underline{\boldsymbol{A}}, \underline{\boldsymbol{A}}^{\prime}\right]=\left\langle\boldsymbol{A}_{1}, \ldots, \boldsymbol{A}_{n}, \boldsymbol{A}_{1}^{\prime}, \ldots, \boldsymbol{A}_{n^{\prime}}^{\prime}\right\rangle,} \\
\left.\left[\underline{\boldsymbol{A}}, \underline{\boldsymbol{A}^{\prime}}\right]\right]=\left[\boldsymbol{A}_{1}, \ldots, \boldsymbol{A}_{n}, \boldsymbol{A}_{1}^{\prime}, \ldots, \boldsymbol{A}_{n^{\prime}}^{\prime}\right]
\end{gathered}
$$

and so on.

The expression (1) is called a representation of $(S)$ of type $(n, k)$. Obviously the h.m.a. having a representation of type $(n, k), k=1,2, \ldots$ for fixed $n$ form a subcategory Set-Mauth $\vec{n}$ of Set-Mauth $\overrightarrow{\text {. }}$.

In Set-Mauth $\overrightarrow{1}$ we define a new kind of parallel connection, which is only a partial binary nondeterministic operation and which will be denoted by $[x]$. Let be given representations

(9) $\quad(S)=\left\langle\boldsymbol{A},\langle\boldsymbol{A}\rangle,\langle\boldsymbol{R}\rangle,(\times \boldsymbol{R}) / \lambda_{\boldsymbol{R}} \underset{l_{, m}}{\stackrel{S}{\rightarrow}} \times \boldsymbol{B}, \underline{\boldsymbol{B}},[\underline{\boldsymbol{B}}]\right\rangle: \boldsymbol{A} \rightarrow[\underline{\boldsymbol{B}}]$,

(10) $\left.\quad\left(S^{\prime}\right)=\left\langle\boldsymbol{A}^{\prime},\left\langle\boldsymbol{A}^{\prime}\right\rangle,\left\langle\boldsymbol{R}^{\prime}\right\rangle,\left(\times \boldsymbol{R}^{\prime}\right) / \lambda_{\boldsymbol{R}^{\prime}} \frac{S^{\prime}}{l^{\prime}, m^{\prime}}\right\rangle \times \boldsymbol{B}^{\prime}, \underline{\boldsymbol{B}}^{\prime},\left[\underline{\boldsymbol{B}}^{\prime}\right]\right\rangle: \boldsymbol{A}^{\prime} \rightarrow\left[\underline{\boldsymbol{B}}^{\prime}\right]$

of two h.m.a. of type $(1, k)$ and $\left(1, k^{\prime}\right)$ respectively. Then the "paralleloidal" connection $(S)[\times]\left(S^{\prime}\right)$ is defined if

$$
\begin{aligned}
& E_{\left[\boldsymbol{A}, A^{\prime}\right]}=E_{\left[\boldsymbol{R}, \boldsymbol{R}^{\prime}\right]}, \\
& \lambda_{\boldsymbol{R}} \times \lambda_{\boldsymbol{R}^{\prime}}=\lambda_{\left[\boldsymbol{R}, \boldsymbol{R}^{\prime}\right]} .
\end{aligned}
$$


If this is the case then $(S)[\times]\left(S^{\prime}\right)$ is a h.m.a. which is given by the representation of type $\left(1, k+k^{\prime}\right)$

(13) $\left(S^{\prime}\right)[\times]\left(S^{\prime}\right)=\left\langle\left[\boldsymbol{A}, \boldsymbol{A}^{\prime}\right],\left\langle\left[\boldsymbol{A}, \boldsymbol{A}^{\prime}\right]\right\rangle,\left\langle\left[\boldsymbol{R}, \boldsymbol{R}^{\prime}\right]\right\rangle\right.$,

$$
\begin{aligned}
\left((\times \boldsymbol{R}) / \lambda_{\boldsymbol{R}} \underset{l, m}{\stackrel{s}{\longrightarrow}} \times \boldsymbol{B}\right) \times\left(\left(\times \boldsymbol{R}^{\prime}\right) / \lambda_{\boldsymbol{R}^{\prime}} \underset{l^{\prime}, m^{\prime}}{s^{\prime}} \times \times \boldsymbol{B}^{\prime}\right), \\
{\left.\left[\underline{\boldsymbol{B}}, \underline{\boldsymbol{B}^{\prime}}\right],\left[\left[\underline{\boldsymbol{B}}, \underline{\boldsymbol{B}^{\prime}}\right]\right]\right\rangle:\left[\boldsymbol{A}, \boldsymbol{A}^{\prime}\right] \rightarrow\left[\left[\underline{\boldsymbol{B}}, \underline{\boldsymbol{B}^{\prime}}\right]\right] . }
\end{aligned}
$$

From (12) it follows the equality

(14) $\quad\left((\times \boldsymbol{R}) / \lambda_{\boldsymbol{R}}\right) \times\left(\left(\times \boldsymbol{R}^{\prime}\right) / \lambda_{\boldsymbol{R}^{\prime}}\right)=\left(\times\left[\boldsymbol{R}, \boldsymbol{R}^{\prime}\right]\right) / \lambda_{\left[\boldsymbol{R}, \boldsymbol{R}^{\prime}\right]}$

and

$$
\begin{aligned}
\left((\times \boldsymbol{R}) / \lambda_{\boldsymbol{R}} \underset{l, m}{\stackrel{S}{\longrightarrow}} \times \boldsymbol{B}\right) \times\left(\left(\times \boldsymbol{R}^{\prime}\right) / \lambda_{\boldsymbol{R}^{\prime}} \frac{S^{\prime}}{l^{\prime}, m^{\prime}} \times \boldsymbol{B}^{\prime}\right) \\
\quad=\left(\times\left[\boldsymbol{R}, \boldsymbol{R}^{\prime}\right]\right) / \lambda_{\left[\boldsymbol{R}, \boldsymbol{R}^{\prime}\right]} \frac{S \times S^{\prime \prime}}{l^{\prime \prime}, m^{\prime \prime}} \times\left[\boldsymbol{B}, \boldsymbol{B}^{\prime}\right]
\end{aligned}
$$

is the ordinary parallel connection of homogeneous monoid automata. Since paralleloidal connection of h.m.a. is not compatible with equality of h.m.a. it is a nondeterministic operation.

\section{THEOREM 4.2. The instruction}

prescribed by

$$
\text { Fh: Set-Auth } \rightarrow \text { Set-Mauth } \overrightarrow{1}
$$

$$
\begin{aligned}
\langle\boldsymbol{X}, \times \boldsymbol{X} \underset{l, m}{\stackrel{s}{\longrightarrow}} & \times \boldsymbol{Y}, \boldsymbol{Y}\rangle \\
& \mapsto\left\langle\boldsymbol{X}^{*},\left\langle\boldsymbol{X}^{*}\right\rangle,\left\langle\left\langle(\times \boldsymbol{X})^{*}\right\rangle\right\rangle, F(\times \boldsymbol{X} \underset{l, m}{\stackrel{s}{\longrightarrow}} \times \boldsymbol{Y}),\left\langle\boldsymbol{Y}^{*}\right\rangle, \boldsymbol{Y}^{*}\right\rangle
\end{aligned}
$$

carries over parallel connection to paralletoidal connection.

Proof. Let be given two h.s.a.

$$
\begin{gathered}
(S)=\langle\boldsymbol{X}, \times \boldsymbol{X} \underset{l, m}{\stackrel{S}{\longrightarrow}} \times \boldsymbol{Y}, \boldsymbol{Y}\rangle, \\
\left(\boldsymbol{S}^{\prime}\right)=\left\langle\boldsymbol{X}^{\prime}, \times \boldsymbol{X}^{\prime} \underset{l^{\prime}, m^{\prime}}{\frac{S^{\prime}}{\longrightarrow}} \times \boldsymbol{Y}^{\prime}, \boldsymbol{Y}^{\prime}\right\rangle .
\end{gathered}
$$

Then one has the formulas

$$
\begin{aligned}
& (S) \times\left(S^{\prime}\right)=\left\langle\left[\boldsymbol{X}, \boldsymbol{X}^{\prime}\right],(\times \boldsymbol{X} \underset{l, m}{\stackrel{s}{\longrightarrow}} \times \boldsymbol{Y}) \times\left(\times \boldsymbol{X}^{\prime} \underset{l^{\prime}, m^{\prime}}{\stackrel{s^{\prime}}{\longrightarrow}} \times \boldsymbol{Y}^{\prime}\right),\left[\boldsymbol{Y}, \boldsymbol{Y}^{\prime}\right]\right\rangle, \\
& \operatorname{Fh}(\boldsymbol{S})=\left\langle\boldsymbol{X}^{*},\left\langle\boldsymbol{X}^{*}\right\rangle,\left\langle\left\langle(\times \boldsymbol{X})^{*}\right\rangle\right\rangle, F\left(\times \boldsymbol{X}_{l, m}^{\stackrel{S}{\longrightarrow}} \times \boldsymbol{Y}\right),\left\langle\boldsymbol{Y}^{*}\right\rangle, \boldsymbol{Y}^{*}\right\rangle, \\
& \operatorname{Fh}\left(S^{\prime}\right)=\left\langle\boldsymbol{X}^{\prime *},\left\langle\boldsymbol{X}^{\prime *}\right\rangle,\left\langle\left\langle\left(\times \boldsymbol{X}^{\prime}\right)^{*}\right\rangle\right\rangle, F^{\prime}\left(\times \boldsymbol{X}^{\prime} \frac{s^{\prime}}{l^{\prime}, m^{\prime}} \times \boldsymbol{Y}^{\prime}\right),\left\langle\boldsymbol{X}^{\prime *}\right\rangle, \boldsymbol{Y}^{\prime *}\right\rangle, \\
& \text { (15) } \operatorname{Fh}\left((S) \times\left(S^{\prime}\right)\right)=\left\langle\left[\boldsymbol{X}^{*}, \boldsymbol{X}^{* *}\right],\left\langle\left[\boldsymbol{X}^{*}, \boldsymbol{X}^{\prime *}\right]\right\rangle,\left\langle\left\langle\left(\times\left[\boldsymbol{X}, \boldsymbol{X}^{\prime}\right]\right)^{*}\right\rangle\right\rangle\right. \text {, } \\
& \left.F^{\prime}\left(\left(\times \boldsymbol{X}_{l, m}^{\stackrel{S}{\longrightarrow}} \times \boldsymbol{Y}\right) \times\left(\times \boldsymbol{X}_{i l^{\prime}, m^{\prime}} \underset{S^{\prime}}{\longrightarrow} \times \boldsymbol{Y}^{\prime}\right)\right),\left\langle\left[\boldsymbol{Y}^{*}, \boldsymbol{Y}^{\prime *}\right]\right\rangle,\left[\boldsymbol{Y}^{*}, \boldsymbol{Y}^{\prime *}\right]\right\rangle .
\end{aligned}
$$

Moreover, it exists

(16) $\operatorname{Fh}\left(S^{\prime}\right)[\times] \operatorname{Fh}\left(S^{\prime}\right)=\left\langle\left[\boldsymbol{X}^{*}, \boldsymbol{X}^{\prime *}\right],\left\langle\left[\boldsymbol{X}^{*}, \boldsymbol{X}^{\prime *}\right]\right\rangle,\left\langle\left\langle(\times \boldsymbol{X})^{*},\left(\times \boldsymbol{X}^{\prime}\right)^{*}\right\rangle\right\rangle\right.$,

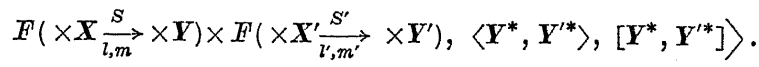

Indeed, E3 implies

$$
E_{\left\langle(\times \boldsymbol{X})^{*},\left(\times \boldsymbol{X}^{\prime}\right)^{*}\right\rangle}=\left(\times \boldsymbol{X} \times \times \boldsymbol{X}^{\prime}\right)^{*}=\left(\times\left[\boldsymbol{X}, \boldsymbol{X}^{\prime}\right]\right)^{*}=E_{\left[\boldsymbol{X}^{*}, \boldsymbol{X}^{*}\right]},
$$

i.e., (11) is true.

${ }_{\nu 2}$ implies $\lambda_{\left\langle(\times \boldsymbol{X})^{*}\right\rangle}=\lambda_{\left\langle\left(\times \boldsymbol{X}^{\prime}\right)^{*}\right\rangle}=0$ (the least congruence),

$\nu 1$ and $\nu 3$ imply $\lambda_{\left\langle(\times X)^{*},\left(\times X^{\prime}\right)^{*}\right\rangle}=0$; hence (12) is true. We claim that (15) and (16) are equal. The verification of this assertion rests upon Lemma 1.2 which implies the equality

$$
\begin{gathered}
\varepsilon\left(\left[\boldsymbol{X}, \boldsymbol{X}^{\prime}\right]\right) F\left((\times \boldsymbol{X} \underset{l, m}{\stackrel{S}{\longrightarrow}} \times \boldsymbol{Y}) \times\left(\times \boldsymbol{X}^{\prime} \underset{l^{\prime} m^{\prime}}{\stackrel{S^{\prime}}{\longrightarrow}} \times \boldsymbol{Y}^{\prime}\right)\right) \mu\left(\times \boldsymbol{Y}, \times \boldsymbol{Y}^{\prime}\right)\left(\mu(\boldsymbol{Y}) \times \mu\left(\boldsymbol{Y}^{\prime}\right)\right) \\
=\varepsilon\left(\left[\boldsymbol{X}, \boldsymbol{X}^{\prime}\right]\right) \mu\left(\times \boldsymbol{X}, \times \boldsymbol{X}^{\prime}\right)\left(F\left(\times \boldsymbol{X}_{l, m}^{\frac{S}{\longrightarrow}} \times \mathbf{Y}\right) \times F\left(\times \boldsymbol{X}^{\prime} \underset{l^{\prime}, m^{\prime}}{\longrightarrow} \times \boldsymbol{Y}^{\prime}\right)\right)(\mu(\boldsymbol{Y}) \times \\
\left.\times \mu\left(\boldsymbol{Y}^{\prime}\right)\right) .
\end{gathered}
$$

The assertion now follows from the equalities

and (cf. $v 3$ )

$$
\mu\left(\times \boldsymbol{Y}, \times \boldsymbol{Y}^{\prime}\right)\left(\mu(\boldsymbol{Y}) \times \mu\left(\boldsymbol{Y}^{\prime}\right)\right)=\mu\left(\left[\boldsymbol{Y}, \mathbf{Y}^{\prime}\right]\right)
$$

$\mu\left(\times X, \times X^{\prime}\right)=v\left(\left\langle(\times X)^{*},\left(\times X^{\prime}\right)^{*}\right\rangle\right)$.

\section{Heterogeneous admissible bisystems}

A heterogeneous admissible bisystem (h.a.b.) is a sequence of the form

where

$$
\begin{gathered}
\underline{\boldsymbol{A}}=\left\langle\boldsymbol{A}_{1}, \ldots, \boldsymbol{A}_{n}\right\rangle, \quad \underline{\boldsymbol{R}}=\left\langle\boldsymbol{R}_{1}, \ldots, \boldsymbol{R}_{n}\right\rangle, \\
\boldsymbol{R}=\left\langle R_{1}, \ldots, R_{n}\right\rangle, \quad R_{i}=\left(\times \boldsymbol{R}_{i}\right) / \lambda_{\boldsymbol{R}_{i}}, \quad E_{\boldsymbol{A}_{i}}=E_{\boldsymbol{B}_{i}}, \\
\underline{\boldsymbol{B}}=\left\langle\boldsymbol{B}_{1}, \ldots, \boldsymbol{B}_{k}\right\rangle, \quad \boldsymbol{B}=\left\langle B_{1}, \ldots, B_{k}\right\rangle, \quad B_{j}=M_{\boldsymbol{B}_{j}}, \quad j=1, \ldots, k,
\end{gathered}
$$

and where $\times \boldsymbol{R} \stackrel{\times \boldsymbol{B}, \boldsymbol{S}}{\longrightarrow} \times \boldsymbol{B}$ is an ordinary (= homogeneous) admissible bisystem.

Two h.a.b. (1) and

(2) $\left\langle\left[\underline{\boldsymbol{A}^{\prime}}\right], \underline{\boldsymbol{A}}^{\prime}, \underline{\boldsymbol{R}^{\prime}}, \times \boldsymbol{R}^{\prime} \stackrel{\boldsymbol{B}^{\prime}, S^{\prime}}{\longrightarrow} \times \boldsymbol{B}, \underline{\boldsymbol{B}^{\prime}},\left[\underline{\boldsymbol{B}^{\prime}}\right]\right\rangle:\left[\underline{\boldsymbol{A}^{\prime}}\right] \rightarrow\left[\underline{\boldsymbol{B}^{\prime}}\right]$ 
are said to be equal, if

$$
\text { (3) }[\underline{A}]=\left[\underline{A}^{\prime}\right], \quad[\underline{B}]=\left[\underline{B}^{\prime}\right] \text {, }
$$

(4) condition 4. (4) is satisfied, where

$$
\begin{aligned}
& \times \boldsymbol{R} \underset{l, m}{\stackrel{S}{\longrightarrow}} \times \boldsymbol{B}=G^{-1}(\times \boldsymbol{R} \stackrel{\times \boldsymbol{B}, S}{\longrightarrow} \times \boldsymbol{B}), \\
& \times \boldsymbol{R}^{\prime} \stackrel{S^{\prime}}{l^{\prime}, m^{\prime}} \times \boldsymbol{B}^{\prime}=G^{-1}\left(\times \boldsymbol{R}^{\prime} \stackrel{\times \boldsymbol{B}^{\prime}, S^{\prime}}{\longrightarrow} \times \boldsymbol{B}^{\prime}\right)
\end{aligned}
$$

are the homogeneous monoid automata which by Theorem 2.1 correspond to the homogeneous admissible bisystems occurring as the arguments of $G^{-1}$. Composition and parallel connection of h.a.b. can be defined in accordance with composition and parallel connection of h.m.a. (applying $G$ to h.m.a. occurring in the sequences (7) and (8)). In analogy with Theorems $2.1,2.2,2.3$ we get

LEMMA 5.1. With respect to the composition just defined the h.a.b. in Set form a category Set-Abih $\rightarrow$. There is a functorial isomorphy

given by

$$
\text { Gh: Set-Mauth } \rightarrow \text { Set-Abih } \rightarrow
$$

$$
\begin{aligned}
&\langle[\underline{\boldsymbol{A}}], \underline{\boldsymbol{A}}, \underline{\boldsymbol{R}}, \times \boldsymbol{R} \underset{l, m}{\stackrel{s}{\longrightarrow}} \times \boldsymbol{B}, \underline{\boldsymbol{B}}, \underline{[\boldsymbol{B}]\rangle} \\
& \mapsto\langle[\underline{\boldsymbol{A}}], \underline{\boldsymbol{A}}, \underline{\boldsymbol{R}}, G(\times \boldsymbol{R} \underset{l, m}{\stackrel{s}{\longrightarrow}} \times \boldsymbol{B}), \underline{\boldsymbol{B}},[\underline{\boldsymbol{B}}]\rangle
\end{aligned}
$$

which is compatible with parallel connection.

We are now in the position to establish the instruction

$$
\text { Set-Auth } \rightarrow \underset{\text { Fh }}{\rightarrow} \text { Set-Mauth } \rightarrow \underset{\text { Gh }}{\rightarrow} \text { Set-Abih } \rightarrow
$$

and call $(\mathrm{Fh} G \mathrm{Gh})(\langle\boldsymbol{X}, \times \boldsymbol{X} \underset{l, m}{\vec{s}} \times \mathbf{Y}, \boldsymbol{Y}\rangle)$ the heterogeneous characteristic bisystem of the h.s.a. $\langle\boldsymbol{X}, \times \boldsymbol{X} \underset{l, m}{\mathrm{~s}} \times \boldsymbol{Y}, \boldsymbol{Y}\rangle$. Its behaviour at least with respect to a suitable defined modified parallel connection (e.g. paralleloidal connection) seems to be a little more convenient than the ordinary (= homogeneous) characteristic bisystem.

\section{References}

[1] E. S. Bainbridge, Feedback and generalized logic, Information and Control 31 (1976), 75-96.

[2] L. Budach und H.-J. Hoehnke, Automaten und Funltoren, Berlin 1975.

\section{Presented to the Semester}

Universal Algebra and Applications (February 15-June 9, 1978)

\section{WARSAW 1982}

\section{REGULARITY IN $p$-ALGEBRAS AND $p$-SEMILATTICES}

J. C. VARLET

University of Liège, Liège, Belgium

\section{Introduction}

An algebra is called regular if any two of its congruences having a class in common are equal. It is well known that a $p$-algebra is regular if and only if it is Boolean. A similar property holds for $p$-semilattices, implicative semilattices and Heyting algebras. Therefore it might seem uninteresting to focus our attention on the concept of regularity when dealing with the aforementioned classes of algebras. However, if one defines regularity not only for classes but also for subsets, congruences and algebras, it appears that $p$-algebras and related structures constitute a nice domain for investigating the notion of regularity. We consider the following three problems :

Problem 1. How to detect a regular congruence?

Problein 2. Where are the regular congruences located in the congruence lattice?

Problem 3. What are the extremely irregular algebras, that is, the algebras in which the universal congruence $\iota$ is the only regular one?

We have a very satisfactory solution of the first problem when the $p$-semilattices and $p$-algebras $L$ are distributive: a congruence $\Theta$ of $L$ is regular if and only if $I_{*} \supseteq D(L)$, where $I=\operatorname{ker} \Theta, I_{*}=\left\{x \in L: x \geqslant i^{*}\right.$ for an $i \in I\}$ and $D(L)$ is the dense set of $L$.

The second problem is answered without any restriction: for all the algebras we deal with, the regular congruences form an increasing subset of $\operatorname{Con}(L)$ contained in $\left[\Theta_{\mathscr{B}}\right)$, where $\Theta_{\mathscr{O}}$ denotes the Glivenko congruence.

Finally, under an assumption of distributivity somewhat slighter than in Problem 1, we show that extreme irregularity is equivalent to any of the following conditions: no *-maximal filter is maximal; every minimal prime ideal $I$ satisfies $I_{*} \subset L-I$; every 2-class congruence covers a 3-class congruence. 